\title{
Dichotomous Hamiltonians with unbounded entries and solutions of Riccati equations
}

\author{
Christiane TRETTER AND CHRISTIAN WYSS
}

Dedicated to Rien Kaashoek on the occasion of his 75th birthday

Abstract. An operator Riccati equation from systems theory is considered in the case that all entries of the associated Hamiltonian are unbounded. Using a certain dichotomy property of the Hamiltonian and its symmetry with respect to two different indefinite inner products, we prove the existence of nonnegative and nonpositive solutions of the Riccati equation. Moreover, conditions for the boundedness and uniqueness of these solutions are established.

\section{Introduction}

In this paper, we prove the existence of solutions of algebraic Riccati equations

$$
A^{*} X+X A+X B X-C=0
$$

on a Hilbert space $H$ where all coefficients are unbounded linear operators and $B, C$ are nonnegative. Riccati equations of this type, and in particular their nonnegative solutions, are of central importance in systems theory, see e.g. [13,22] and the references therein; recently, the case of unbounded $B$ and $C$ has gained much attention $[26,31,32,39]$.

The existence of solutions $X$ of the Riccati equation (1) is intimately related to the existence of graph subspaces $G(X)=\{(u, X u) \mid u \in \mathcal{D}(X)\}$ that are invariant under the associated Hamiltonian

$$
T=\left(\begin{array}{cc}
A & B \\
C & -A^{*}
\end{array}\right) .
$$

Moreover, properties of a solution $X$ of (1) such as selfadjointness, nonnegativity, or boundedness can be characterised by properties of the corresponding graph subspace $G(X)$ with respect to certain indefinite inner products.

Mathematics Subject Classification: 47A62, 47B44, 47N70.

Keywords: Riccati equation, Hamiltonian, dichotomous, bisectorial, invariant subspace, $p$-subordinate perturbation. 
In the finite-dimensional case, the connection between solutions of Riccati equations and invariant graph subspaces of Hamiltonians led to an extensive description of all solutions, see e.g. [10,22]. In the infinite-dimensional case, the existence of invariant subspaces is a more subtle problem since the Hamiltonian $T$ is not normal. If all coefficients of the Riccati equation, and hence all entries of $T$, are unbounded, the spectrum of the Hamiltonian may touch at infinity and there are neither the spectral theorem nor Riesz projections available to define invariant subspaces.

There are two different approaches to overcome these difficulties which require different additional properties of the Hamiltonian $T$. In [21,41,42], infinitely many solutions of (1) were constructed in the case that $T$ has a Riesz basis of (possibly generalised) eigenvectors. In [11,24], the existence of a nonnegative and a nonpositive solution and conditions for their boundedness were obtained in the case that $T$ is dichotomous and $B, C$ are bounded.

In the present paper, we prove the existence of solutions of the Riccati equation (1) and characterise their properties without the assumptions that $T$ has a Riesz basis of generalised eigenvectors or that $B, C$ are bounded.

To this end, we follow the dichotomy approach, but essentially new techniques are needed to establish the boundedness of solutions of the Riccati equation in the presence of unbounded $B$ and $C$. In our main result (Theorem 5.3), we show that if $T$ is a nonnegative diagonally $p$-dominant Hamiltonian (i.e. $B, C$ are nonnegative and $p$-subordinate to $A^{*}, A$, respectively, with $p<1$ ), the state operator $A$ is sectorially dichotomous, and $\bigcap_{t \in \mathbb{R}} \operatorname{ker}\left(B\left(A^{*}+\mathrm{i} t\right)^{-1}\right)=\{0\}$, then there exists a nonnegative solution $X_{+}$and a nonpositive solution $X_{-}$of the Riccati equation (1) or, more precisely, of

$$
\left(A^{*} X_{ \pm}+X_{ \pm}\left(A+B X_{ \pm}\right)-C\right) u=0, \quad u \in \mathcal{D}(A) \cap X_{ \pm}^{-1} \mathcal{D}\left(A^{*}\right) .
$$

In our second main result (Theorem 6.4), we show that if e.g. $A$ is sectorial with angle $\theta<\pi / 2$, then the nonnegative solution $X_{+}$is bounded, uniquely determined and (3) holds for all $u \in \mathcal{D}(A)$; similarly, if $-A$ is sectorial with angle $\theta<\pi / 2$, then $X_{-}$is bounded and uniquely determined.

The assumption $\bigcap_{t \in \mathbb{R}} \operatorname{ker}\left(B\left(A^{*}+\mathrm{i} t\right)^{-1}\right)=\{0\}$ is trivially satisfied if $\operatorname{ker} B=\{0\}$. A necessary condition for it is that $\operatorname{ker} B$ contains no eigenvectors of $A^{*}$; if $A$ has a compact resolvent and the system of generalised eigenvectors is complete, it is also sufficient. If $A$ generates a $C_{0}$-semigroup and $B$ is bounded, it is equivalent to the approximate controllability of the pair $(A, B)$.

A novel ingredient of our approach is stability theorems for $p$-subordinate perturbations of sectorially dichotomous operators. In brief, a linear operator $R$ on a Banach space $V$ is called $p$-subordinate to a linear operator $S$ on $V$ with $p \in[0,1]$ if $\mathcal{D}(S) \subset \mathcal{D}(R)$ and there exists $c \geq 0$ with

$$
\|R u\| \leq c\|u\|^{1-p}\|S u\|^{p}, \quad u \in \mathcal{D}(S) ;
$$

if $p<1$, this implies that $R$ is $S$-bounded with $S$-bound 0 . A linear operator $S$ on $V$ is called dichotomous if the spectrum $\sigma(S)$ has a gap along the imaginary axis $i \mathbb{R}$ 
and there is a decomposition $V=V_{+} \oplus V_{-}$into $S$-invariant subspaces $V_{ \pm}$such that the restrictions $S_{+}=\left.S\right|_{V_{+}}$and $S_{-}=\left.S\right|_{V_{-}}$have their spectrum in the right and left half-plane, respectively; note that, even in the Hilbert space case, orthogonality is not assumed. If $-S_{+}$and $S_{-}$are generators of exponentially decaying semigroups, then $S$ is called exponentially dichotomous, see [9]; if these semigroups are even analytic, then $S$ is sectorially dichotomous, see Sect. 2 below.

The assumption that the state operator $A$ is sectorially dichotomous implies that $A$ is bisectorial (i.e. a bisector around $\mathrm{i} \mathbb{R}$ is contained in the resolvent set $\varrho(A)$ and $\lambda(A-\lambda)^{-1}$ is uniformly bounded on this bisector). Bisectorial operators play an important role in the study of maximal regularity of evolution equations $u^{\prime}+A u=f$ on $\mathbb{R}$, see e.g. $[4,5]$. Exponentially dichotomous operators have a wide range of applications, e.g. to Wiener-Hopf factorisation, see $[8,9,37]$. The spectral decomposition of a dichotomous Hamiltonian operator function may be used to show the conditional reducibility of this operator function, see [6].

In systems theory, e.g. for systems with boundary control and observation, see [42], the unbounded operators $B$ and $C$ need not have realisations as symmetric operators on $H$ but, instead, map into an extrapolation space. The results of this paper are a first step in this direction; the generalisation to Riccati equations involving extrapolation spaces is work in progress.

The article is organised as follows: In Sect. 2, we introduce sectorially dichotomous operators and present some of their important properties. In Sect. 3, we study the stability of bisectoriality and sectorial dichotomy under $p$-subordinate perturbations, and we investigate their effect on the spectrum. In Sect. 4, we prove that a Hamiltonian (2) with sectorially dichotomous $A$ and nonnegative $B, C$ that are $p$-subordinate to $A^{*}, A$, respectively, is dichotomous. We employ the symmetry of $T$ with respect to two different indefinite inner products $[\cdot \mid \cdot]_{1},[\cdot \mid \cdot]_{2}$, used before in $[21,23,24]$, to show that the corresponding invariant subspaces $V_{+}, V_{-}$are hypermaximal neutral in $[\cdot \mid \cdot]_{1}$ and nonnegative, nonpositive, respectively, in $[\cdot \mid \cdot]_{2}$. In Sect. 5, we exploit these properties to prove, in Theorem 5.3, that $V_{ \pm}$are graphs or inverse graphs of operators $X_{ \pm}$ and that $X_{ \pm}$are solutions of the Riccati equation (1) if $\bigcap_{t \in \mathbb{R}} \operatorname{ker}\left(B\left(A^{*}+\mathrm{i} t\right)^{-1}\right)=\{0\}$. Moreover, we derive necessary as well as sufficient conditions for the latter assumption. In Sect. 6, we prove, in Theorem 6.4, that $X_{+}$(or $X_{-}$) is bounded and uniquely determined provided that $A$ (or $-A$ ) is sectorial with angle $\theta<\pi / 2$. Our proof exploits the continuous dependence of the subspaces $V_{ \pm}$, and hence of $X_{ \pm}$, on $B$ and $C$, see Proposition 6.3; it differs substantially from the one in [24] for bounded $B, C$. In the final Sect. 7, we illustrate our theory by three examples in which all entries of the Hamiltonian are partial differential or unbounded multiplication operators; in all cases, neither the results of $[11,24]$ nor those of $[21,41,42]$ apply, either because $B, C$ are unbounded or because the Hamiltonian does not have a Riesz basis of generalised eigenvectors.

In this paper, the following notation is used. For a closed linear operator $T$ on a Banach space $V$, we denote the domain by $\mathcal{D}(T)$, the kernel by $\operatorname{ker}(T)$, the spectrum 
by $\sigma(T)$, the point spectrum by $\sigma_{p}(T)$, and the resolvent set by $\varrho(T)$. Further, by $\mathbb{C}_{+}$ and $\mathbb{C}_{-}$, we denote the open right and open left half-plane, respectively.

\section{Sectorially dichotomous operators}

In this section, we introduce and study sectorially dichotomous operators. They form a subclass of exponentially dichotomous operators for which there exist invariant spectral subspaces corresponding to the spectral parts in the left and the right halfplane, even if none of them is bounded.

We begin by briefly recalling the notions of dichotomous and exponentially dichotomous operators, see [9,24], and of sectorial and bisectorial operators, see [4].

DEFINITION 2.1. A densely defined linear operator $S$ on a Banach space $V$ is called dichotomous if there exist $h>0$ and complementary closed subspaces $V_{+}, V_{-} \subset V$, i.e. $V=V_{+} \oplus V_{-}$, such that

(i) $\{z \in \mathbb{C}|| \operatorname{Re} z \mid<h\} \subset \varrho(S)$,

(ii) $V_{+}$and $V_{-}$are $S$-invariant, i.e. $S\left(\mathcal{D}(S) \cap V_{ \pm}\right) \subset V_{ \pm}$, and

(iii) $\sigma\left(\left.S\right|_{V_{+}}\right) \subset \mathbb{C}_{+}$and $\sigma\left(\left.S\right|_{V_{-}}\right) \subset \mathbb{C}_{-}$;

in this case, the maximal $h_{0}$ with (i) is called dichotomy gap of $S$. A dichotomous operator is called exponentially dichotomous if

(iv) $-\left.S\right|_{V_{+}}$and $\left.S\right|_{V_{-}}$are generators of exponentially decaying semigroups.

We call $V_{ \pm}$the spectral subspaces corresponding to the dichotomous operator $S$; we write $S_{ \pm}:=\left.S\right|_{V_{ \pm}}$for the restrictions of $S$ to $V_{ \pm}$and denote by $P_{ \pm}$the spectral projections onto $V_{ \pm}$.

Dichotomous operators admit a block diagonal matrix representation with respect to the decomposition $V=V_{+} \oplus V_{-}$in the following sense:

DEFINITION 2.2. ([17, §III.5.6]) Let $S$ be a linear operator on a Banach space $V$ and $V_{1}, V_{2} \subset V$ complementary closed subspaces. Then $S$ is said to decompose with respect to the direct sum $V=V_{1} \oplus V_{2}$ if

(i) $V_{1}$ and $V_{2}$ are $S$-invariant, and

(ii) $\mathcal{D}(S)=\left(\mathcal{D}(S) \cap V_{1}\right) \oplus\left(\mathcal{D}(S) \cap V_{2}\right)$.

Note that even in the Hilbert space case, it is not assumed that $V_{1}$ and $V_{2}$ are orthogonal, i.e. $V_{1}$ is not a reducing subspace of $S$ in the sense of $[3,38]$.

REMARK 2.3. If $S$ decomposes with respect to $V=V_{1} \oplus V_{2}$, then $S$ admits the block operator matrix representation

$$
S=\left(\begin{array}{cc}
\left.S\right|_{V_{1}} & 0 \\
0 & \left.S\right|_{V_{2}}
\end{array}\right)
$$

in particular, $\sigma(S)=\sigma\left(\left.S\right|_{V_{1}}\right) \cup \sigma\left(\left.S\right|_{V_{2}}\right)$ and, for every $z \in \varrho(S)$, the subspaces $V_{1}$ and $V_{2}$ are also $(S-z)^{-1}$-invariant. 
LEMMA 2.4. If the linear operator $S$ is dichotomous, then it decomposes with respect to its spectral subspaces $V=V_{+} \oplus V_{-}$.

Proof. We only have to verify property (ii) in Definition 2.2. The inclusion " $\supset$ " is trivial. Let $x \in \mathcal{D}(S)$. Then $S x=y_{+}+y_{-}$with $y_{ \pm} \in V_{ \pm}$. Since $0 \in \varrho\left(S_{ \pm}\right)$by condition (iii) in Definition 2.1, we can set $x_{ \pm}:=\left(S_{ \pm}\right)^{-1} y_{ \pm} \in \mathcal{D}(S) \cap V_{ \pm}$and obtain

$$
S\left(x_{+}+x_{-}\right)=S_{+} x_{+}+S_{-} x_{-}=y_{+}+y_{-}=S x .
$$

Because $0 \in \varrho(S)$, this implies that $x=x_{+}+x_{-} \in\left(\mathcal{D}(S) \cap V_{+}\right) \oplus\left(\mathcal{D}(S) \cap V_{-}\right)$.

REMARK 2.5. There are two simple cases in which condition (i) in Definition 2.1, $\{z \in \mathbb{C}|| \operatorname{Re} z \mid<h\} \subset \varrho(S)$, already suffices for the dichotomy of $S$ :

1. if $S$ is a normal operator on a Hilbert space;

2. if one of $\sigma_{ \pm}(S)=\sigma(S) \cap \mathbb{C}_{ \pm}$is bounded.

In the first case, the existence of the subspaces $V_{ \pm}$is a consequence of the spectral theorem; in the second case, the Riesz projection corresponding to the bounded part $\sigma_{-}(S)$ or $\sigma_{+}(S)$ of $\sigma(S)$ may be used to define $V_{-}$or $V_{+}$, compare [17, §III.6.4].

The following result is essential in characterising dichotomous operators possessing the additional property that the spectral projections are given by a resolvent integral along the imaginary axis; its proof is based on an earlier deep result by Bart, Gohberg, and Kaashoek, see [9, Theorem 3.1] and also [16, Theorem XV.3.1].

THEOREM 2.6. ([25, Theorem 1.1]) Let $S$ be a closed densely defined linear operator on a Banach space $V$ and $h>0$ such that

(i) $\{z \in \mathbb{C}|| \operatorname{Re} z \mid \leq h\} \subset \varrho(S)$ and $\sup _{|\operatorname{Re} z| \leq h}\left\|(S-z)^{-1}\right\|<\infty$;

(ii) $\lim _{|s| \rightarrow \infty} \sup _{r \in[0, h]}\left\|(S-r-\mathrm{i} s)^{-1}\right\|=0$;

(iii) the Cauchy principal value at infinity $\int_{\mathrm{i} \mathbb{R}}^{\prime}(S-z)^{-1} x \mathrm{~d} z$ exists for all $x \in V$.

Then $S$ is dichotomous and the corresponding projections $P_{+}, P_{-}$satisfy

$$
\frac{1}{\pi \mathrm{i}} \int_{\mathrm{i} \mathbb{R}}^{\prime}(S-z)^{-1} x \mathrm{~d} z=P_{+} x-P_{-} x, \quad x \in V .
$$

REMARK 2.7. A standard Neumann series argument shows that assumptions (i) and (ii) in Theorem 2.6 are satisfied if

$$
\mathrm{i} \mathbb{R} \subset \varrho(S) \text { and } \lim _{|t| \rightarrow \infty}\left\|(S-\mathrm{i} t)^{-1}\right\|=0
$$

To obtain a sufficient condition for assumption (iii), we now introduce sectorially dichotomous operators, which form a subclass of exponentially dichotomous operators. First, we need the notion of sectorial and bisectorial operators, see e.g. [4].

DEFINITION 2.8. Let $S$ be a densely defined linear operator on a Banach space. 
(i) $S$ is called sectorial with angle $e^{1} \theta \in[0, \pi$ [ and radius $r \geq 0$ if

$$
\sigma(S) \subset \Sigma_{\theta} \cup \overline{B_{r}(0)} \quad \text { where } \quad \Sigma_{\theta}:=\{z \in \mathbb{C}|| \arg z \mid \leq \theta\}
$$

and for every $\left.\left.\theta^{\prime} \in\right] \theta, \pi\right]$ there exists $M>0$ such that

$$
\left\|(S-z)^{-1}\right\| \leq \frac{M}{|z|}, \quad|\arg z| \geq \theta^{\prime},|z|>r ;
$$

$S$ is called sectorial with angle $\theta \in[0, \pi$ [, or simply sectorial, if $r=0$.

(ii) $S$ is called bisectorial with angle $\theta \in[0, \pi / 2$ [ and radius $r \geq 0$ if

$$
\sigma(S) \subset \Sigma_{\theta} \cup\left(-\Sigma_{\theta}\right) \cup \overline{B_{r}(0)}
$$

and for every $\left.\left.\theta^{\prime} \in\right] \theta, \pi / 2\right]$ there exists $M>0$ such that

$$
\left\|(S-z)^{-1}\right\| \leq \frac{M}{|z|}, \quad \theta^{\prime} \leq|\arg z| \leq \pi-\theta^{\prime},|z|>r ;
$$

$S$ is called bisectorial with angle $\theta \in[0, \pi / 2[$, or simply bisectorial, if $r=0$.

The bisector on which the resolvent estimate (6) holds is denoted by, see Fig. 2,

$$
\Omega_{\theta^{\prime}, r}:=\left\{z \in \mathbb{C}\left|\theta^{\prime} \leq\right| \arg z\left|\leq \pi-\theta^{\prime},\right| z \mid>r\right\} .
$$

REMARK 2.9. (i) In semigroup theory, often $-S$ instead of $S$ is called sectorial.

(ii) $S$ is sectorial with angle $\theta<\pi / 2$ if and only if $-S$ is the generator of a bounded analytic semigroup, see e.g. [15, Theorem II.4.6].

(iii) If $V$ is a Hilbert space with scalar product $(\cdot \mid \cdot)$ and

$$
W(S):=\{(S x \mid x) \mid x \in \mathcal{D}(S),\|x\|=1\}
$$

is the numerical range of $S$, then $S$ is sectorial with angle $\theta \leq \pi / 2$ if

$$
W(S) \subset \Sigma_{\theta} \text { and } \varrho(S) \backslash W(S) \neq \varnothing ;
$$

in this case, for every $\left.\left.\theta^{\prime} \in\right] \theta, \theta+\pi / 2\right]$ the resolvent estimate (5) holds with $M=\left(\sin \left(\theta^{\prime}-\theta\right)\right)^{-1}$ and $r=0$, compare [17, Theorem V.3.2 and $\S$ V.3.10].

(iv) If $S$ satisfies (5) for some $\left.\left.\theta^{\prime} \in\right] 0, \pi\right], r \geq 0$ and $M>0$, then there exists $\theta \in\left[0, \theta^{\prime}[\right.$ such that $S$ is sectorial with angle $\theta$ and radius $r$; this follows from a standard Neumann series argument. Similarly, if $S$ satisfies (6) for some $\left.\left.\theta^{\prime} \in\right] 0, \pi / 2\right], r \geq 0$ and $M>0$, then there exists $\theta \in\left[0, \theta^{\prime}[\right.$ such that $S$ is bisectorial with angle $\theta$ and radius $r$.

(v) If $S$ is the direct sum of two operators $S_{+}$and $S_{-}$where $S_{+}$and $-S_{-}$are sectorial with angle $\theta<\pi / 2$ and radius $r \geq 0$, then $S$ is bisectorial with angle $\theta$ and radius $r$, see the proof of Lemma 2.12 (ii) below.

\footnotetext{
${ }^{1}$ Throughout the article we use the conventions $-\pi<\arg z \leq \pi$ and $\arg 0=0$ for the argument of a complex number.
} 


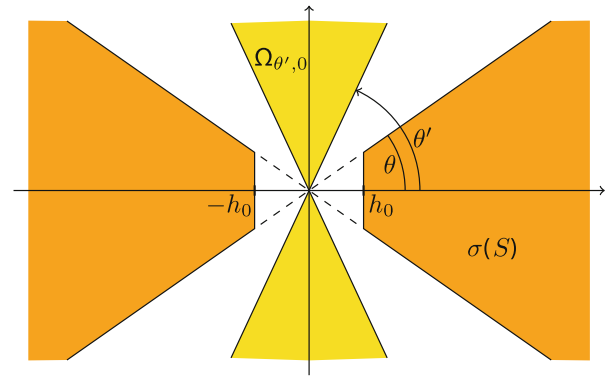

Figure 1. Situation in the proof of Lemma 2.12(ii) for sectorially dichotomous $S$

DEFINITION 2.10. A dichotomous operator $S$ on a Banach space is called sectorially dichotomous with angle $\theta \in\left[0, \pi / 2\left[\right.\right.$ if $S_{+},-S_{-}$are sectorial with angle $\theta$.

REMARK 2.11. (i) The operator $S$ is sectorially dichotomous if and only if it is exponentially dichotomous and the exponentially decaying semigroups generated by $-S_{+}$and $S_{-}$are analytic.

(ii) A simple example for an operator that is exponentially, but not sectorially dichotomous, is a normal operator with discrete spectrum and eigenvalues $1+\mathrm{i} k$ and $-1+\mathrm{i} k, k \in \mathbb{N}$.

The next lemma shows that sectorially dichotomous operators are bisectorial (compare Fig. 1) and satisfy condition (iii) in Theorem 2.6.

LEMMA 2.12. Let $S$ be sectorially dichotomous with angle $\theta \in[0, \pi / 2[$ and dichotomy gap $h_{0}>0$. Then

(i) $\sigma(S) \subset\left\{z \in \Sigma_{\theta} \cup\left(-\Sigma_{\theta}\right)|| \operatorname{Re} z \mid \geq h_{0}\right\}$;

(ii) $S$ is bisectorial with angle $\theta$;

(iii) the spectral projections $P_{+}, P_{-}$corresponding to $S$ satisfy

$$
\frac{1}{\pi \mathrm{i}} \int_{\mathrm{i} \mathbb{R}}^{\prime}(S-z)^{-1} x \mathrm{~d} z=P_{+} x-P_{-} x, \quad x \in V .
$$

Proof. (i) The claim is immediate from Definitions 2.1, 2.8 and 2.10 because $\sigma(S)=\sigma\left(S_{+}\right) \cup \sigma\left(S_{-}\right)$.

(ii) Let $\left.\left.\theta^{\prime} \in\right] \theta, \pi / 2\right]$. Since $S_{+}$and $-S_{-}$are sectorial with angle $\theta$, there exist $M_{ \pm}>0$ such that for $|\arg z| \geq \theta^{\prime}$ we have $\left\|\left( \pm S_{ \pm}-z\right)^{-1}\right\| \leq M_{ \pm} /|z|$. For $z \in \Omega_{\theta^{\prime}, 0}$ we thus obtain, with $M:=M_{+}\left\|P_{+}\right\|+M_{-}\left\|P_{-}\right\|$,

$$
\left\|(S-z)^{-1}\right\| \leq\left\|\left(S_{+}-z\right)^{-1} P_{+}\right\|+\left\|\left(S_{-}-z\right)^{-1} P_{-}\right\| \leq \frac{M}{|z|} .
$$

(iii) Since $S_{+}$and $-S_{-}$are sectorial with angle $\theta<\pi / 2$ and $0 \in \varrho\left(S_{ \pm}\right)$, [24, Lemma 6.1] implies that

$$
\int_{\mathrm{i} \mathbb{R}}^{\prime}\left( \pm S_{ \pm}-z\right)^{-1} x \mathrm{~d} z=\mathrm{i} \pi x, \quad x \in V_{ \pm} .
$$


Consequently,

$$
\begin{aligned}
\int_{\mathrm{i} \mathbb{R}}^{\prime}(S-z)^{-1} x \mathrm{~d} z & =\int_{\mathrm{i} \mathbb{R}}^{\prime}\left(S_{+}-z\right)^{-1} P_{+} x \mathrm{~d} z+\int_{\mathrm{i} \mathbb{R}}^{\prime}\left(S_{-}-z\right)^{-1} P_{-} x \mathrm{~d} z \\
& =\mathrm{i} \pi P_{+} x-\mathrm{i} \pi P_{-} x
\end{aligned}
$$

for all $x \in V$.

REMARK 2.13. Not every bisectorial operator with $0 \in \varrho(S)$ is sectorially dichotomous, see [30, Theorem 3] for a counter-example; note that hence the second implication of [37, Proposition 1.8] does not hold. The question whether a bisectorial and dichotomous operator $S$ is sectorially dichotomous will be considered in a forthcoming paper; while we know that the restrictions $S_{+}$and $-S_{-}$have their spectrum in a sector $\Sigma_{\theta}$ and satisfy resolvent estimates on $\Omega_{\theta, 0}$, it is not clear that these estimates also hold on the left half-plane, as required for sectoriality.

In Sect. 4 below, we will consider Hamiltonians whose state operator $A$ is sectorially dichotomous; in systems theory $A$ is usually even assumed to generate a strongly continuous semigroup. The following lemma characterises this situation.

LEMMA 2.14. For a linear operator $S$ in a Banach space the following are equivalent:

(i) S is sectorially dichotomous and generates a strongly continuous semigroup;

(ii) $S$ is sectorially dichotomous with bounded $S_{+}$;

(iii) $S$ generates a (not necessarily bounded) analytic semigroup and $\mathrm{i} \mathbb{R} \subset \varrho(S)$.

Proof. (i) $\Rightarrow$ (iii): Since $S$ generates a strongly continuous semigroup, there are $M>0$ and $\omega \in \mathbb{R}$ such that

$$
\left\|(S-z)^{-1}\right\| \leq \frac{M}{\operatorname{Re} z-\omega}, \quad \operatorname{Re} z>\omega .
$$

Together with (6), it is not difficult to conclude that there exist $M^{\prime}>0, \omega^{\prime}>\omega$ and $\varphi>\pi / 2$ such that

$$
\left\|(S-z)^{-1}\right\| \leq \frac{M^{\prime}}{\left|z-\omega^{\prime}\right|}, \quad\left|\arg \left(z-\omega^{\prime}\right)\right| \leq \varphi .
$$

Hence $S$ generates an analytic semigroup.

(iii) $\Rightarrow$ (ii): Since $S$ generates an analytic semigroup, it satisfies an estimate (9). Together with the assumption $i \mathbb{R} \subset \varrho(S)$ this implies that the part $\sigma_{+}(S)$ of the spectrum in the right half-plane is bounded and hence $S$ is dichotomous with bounded $S_{+}$, see Remark 2.5; in particular, $S_{+}$is sectorial with angle less than $\pi / 2$. By (9), also $-S_{-}$is sectorial with angle less than $\pi / 2$ and thus $S$ is sectorially dichotomous.

(ii) $\Rightarrow$ (i): Since $S_{+}$is bounded, it generates a strongly continuous semigroup. Due to the sectorial dichotomy, the same is true for $S_{-}$and hence also for $S$. 
Next we show that the adjoint $S^{*}$ of a sectorially dichotomous operator $S$ on a Hilbert space $H$ is again sectorially dichotomous. The difficulty here is that the spectral decomposition $H=H_{+} \oplus H_{-}$of $S$ is not necessarily orthogonal; for the simpler orthogonal case, compare [38, Exercise 5.39].

LEMMA 2.15. Let $S$ be a closed densely defined linear operator on a Hilbert space $H$ that decomposes with respect to a (not necessarily orthogonal) direct sum $H=H_{1} \oplus H_{2}$. Then $S^{*}$ decomposes with respect to $H=H_{2}^{\perp} \oplus H_{1}^{\perp}$, and we have ${ }^{2}$

$$
\sigma\left(\left.S^{*}\right|_{H_{2}^{\perp}}\right)=\sigma\left(\left.S\right|_{H_{1}}\right)^{*}, \quad \sigma\left(\left.S^{*}\right|_{H_{1}^{\perp}}\right)=\sigma\left(\left.S\right|_{H_{2}}\right)^{*}
$$

moreover, if $P_{1 / 2}$ are the projections onto $H_{1 / 2}$ associated with $H=H_{1} \oplus H_{2}$, then

$$
\begin{aligned}
\left\|\left(\left.S^{*}\right|_{H_{2}^{\perp}}-\bar{z}\right)^{-1}\right\| & \leq\left\|P_{1}\right\|\left\|\left(\left.S\right|_{H_{1}}-z\right)^{-1}\right\|, \\
\left\|\left(\left.S^{*}\right|_{H_{1}^{\perp}}-\bar{z}\right)^{-1}\right\| & \leq\left\|P_{2}\right\|\left\|\left(\left.S\right|_{H_{2}}-z\right)^{-1}\right\| .
\end{aligned}
$$

Proof. We have $I=P_{1}+P_{2}$ and $\mathcal{R}\left(P_{j}\right)=H_{j}$. Hence $P_{1}^{*}, P_{2}^{*}$ are projections with $I=P_{1}^{*}+P_{2}^{*}$ and

$$
\mathcal{R}\left(P_{1}^{*}\right)=\operatorname{ker} P_{2}^{*}=\mathcal{R}\left(P_{2}\right)^{\perp}=H_{2}^{\perp}, \quad \mathcal{R}\left(P_{2}^{*}\right)=H_{1}^{\perp} ;
$$

in particular, $H=H_{2}^{\perp} \oplus H_{1}^{\perp}$.

To show that $H_{1}^{\perp}$ is $S^{*}$-invariant, let $y \in H_{1}^{\perp} \cap \mathcal{D}\left(S^{*}\right)$ and $x \in H_{1} \cap \mathcal{D}(S)$. Then $\left(S^{*} y \mid x\right)=(y \mid S x)=0$ since $S x \in H_{1}$. Because $\mathcal{D}(S) \subset H$ is dense, $H_{1} \cap \mathcal{D}(S) \subset H_{1}$ is dense, too, and we obtain $S^{*} y \in H_{1}^{\perp}$. Similarly, one can show that $H_{2}^{\perp}$ is $S^{*}$ invariant. Now let $y \in \mathcal{D}\left(S^{*}\right)$ and $x \in \mathcal{D}(S)$. Since $\left(S P_{1}\right)^{*} \supset P_{1}^{*} S^{*}$ and hence $\left.\left(S P_{1}\right)^{*}\right|_{\mathcal{D}\left(S^{*}\right)}=P_{1}^{*} S^{*}$, we have that

$$
\left(P_{1}^{*} y \mid S x\right)=\left(y \mid P_{1} S x\right)=\left(y \mid S P_{1} x\right)=\left(\left(S P_{1}\right)^{*} x \mid x\right)=\left(P_{1}^{*} S^{*} y \mid x\right)
$$

is bounded in $x$ and thus $P_{1}^{*} y \in \mathcal{D}\left(S^{*}\right)$. This implies that $P_{2}^{*} y=y-P_{1}^{*} y \in \mathcal{D}\left(S^{*}\right)$. Thus

$$
\mathcal{D}\left(S^{*}\right)=\left(\mathcal{D}\left(S^{*}\right) \cap \mathcal{R}\left(P_{1}^{*}\right)\right) \oplus\left(\mathcal{D}\left(S^{*}\right) \cap \mathcal{R}\left(P_{2}^{*}\right)\right),
$$

and so $S^{*}$ decomposes with respect to $H=H_{2}^{\perp} \oplus H_{1}^{\perp}$.

Finally, let $z \in \varrho\left(\left.S\right|_{H_{1}}\right)$ and $R_{1}:=\left(\left.S\right|_{H_{1}}-z\right)^{-1} P_{1}: H \rightarrow H$. Then

$$
\begin{array}{ll}
P_{1} x=R_{1}(S-z) x, & x \in \mathcal{D}(S), \\
P_{1} x=(S-z) R_{1} x, & x \in H .
\end{array}
$$

We have $\left(R_{1}(S-z)\right)^{*}=\left(S^{*}-\bar{z}\right) R_{1}^{*}$ because $R_{1}$ is bounded and $\left.\left((S-z) R_{1}\right)^{*}\right|_{\mathcal{D}\left(S^{*}\right)}=$ $R_{1}^{*}\left(S^{*}-\bar{z}\right)$. Hence we obtain

$$
\begin{array}{ll}
P_{1}^{*} y=\left(S^{*}-\bar{z}\right) R_{1}^{*} y, & y \in H, \\
P_{1}^{*} y=R_{1}^{*}\left(S^{*}-\bar{z}\right) y, & y \in \mathcal{D}\left(S^{*}\right) .
\end{array}
$$

\footnotetext{
${ }^{2}$ We denote the complex conjugate of a set $G \subset \mathbb{C}$ by $G^{*}=\{\bar{z} \in \mathbb{C} \mid z \in G\}$.
} 
Since $\overline{\mathcal{R}\left(R_{1}^{*}\right)}=\left(\operatorname{ker} R_{1}\right)^{\perp}=\left(\operatorname{ker} P_{1}\right)^{\perp}=H_{2}^{\perp}=\mathcal{R}\left(P_{1}^{*}\right)$, this yields

$$
\begin{aligned}
& y=\left(\left.S^{*}\right|_{H_{2}^{\perp}}-\bar{z}\right) R_{1}^{*} y, \quad y \in H_{2}^{\perp}, \\
& y=R_{1}^{*}\left(\left.S^{*}\right|_{H_{2}^{\perp}}-\bar{z}\right) y, \quad y \in \mathcal{D}\left(S^{*}\right) \cap H_{2}^{\perp} .
\end{aligned}
$$

Consequently, $\bar{z} \in \varrho\left(\left.S^{*}\right|_{H_{2}^{\perp}}\right)$ with $\left(\left.S^{*}\right|_{H_{2}^{\perp}}-\bar{z}\right)^{-1}=\left.R_{1}^{*}\right|_{H_{2}^{\perp}}$. Exchanging the roles of $S$ and $S^{*}$ as well as those of $H_{1}$ and $H_{2}$, we obtain $\varrho\left(\left.S\right|_{H_{1 / 2}}\right)=\varrho\left(\left.S^{*}\right|_{H_{2 / 1}^{\perp}}\right)^{*}$ and

$$
\left\|\left(\left.S^{*}\right|_{H_{2 / 1}^{\perp}}-\bar{z}\right)^{-1}\right\| \leq\left\|R_{1 / 2}^{*}\right\|=\left\|R_{1 / 2}\right\| \leq\left\|P_{1 / 2}\right\|\left\|\left(\left.S\right|_{H_{1 / 2}}-z\right)^{-1}\right\| .
$$

COROLLARY 2.16. If $S$ is a sectorially dichotomous operator with angle $\theta \in$ $\left[0, \pi / 2\left[\right.\right.$ on a Hilbert space, then the adjoint $S^{*}$ is also sectorially dichotomous with angle $\theta$.

Proof. Let $H=H_{+} \oplus H_{-}$be the decomposition corresponding to $S$ and $h_{0}>0$ the dichotomy gap of $S$. Then $S^{*}$ decomposes with respect to $H=H_{-}^{\perp} \oplus H_{+}^{\perp}$. Moreover,

$$
\sigma\left(\left.S^{*}\right|_{H_{-}^{\perp}}\right)=\sigma\left(\left.S\right|_{H_{+}}\right)^{*} \subset\left\{z \in \Sigma_{\theta} \mid \operatorname{Re} z \geq h_{0}\right\}
$$

and for $\theta^{\prime}>\theta$ there exists $M>0$ such that

$$
\left\|\left(\left.S^{*}\right|_{H_{-}^{\perp}}-z\right)^{-1}\right\| \leq\left\|P_{+}\right\|\left\|\left(\left.S\right|_{H_{+}}-\bar{z}\right)^{-1}\right\| \leq \frac{M\left\|P_{+}\right\|}{|z|}, \quad|\arg z| \geq \theta^{\prime} .
$$

An analogous reasoning applies to $-\left.S^{*}\right|_{H_{+}^{\perp}}$, and we conclude that $S^{*}$ is sectorially dichotomous with angle $\theta$.

\section{3. $p$-Subordinate perturbations}

In this section, we show that bisectoriality is stable under $p$-subordinate perturbations and that $p$-subordinate perturbations of sectorially dichotomous operators are still dichotomous. To begin with, we briefly recall the concept of $p$-subordinate perturbations which was studied, e.g. in [20, §I.7.1] and [28, §5].

DEFINITION 3.1. Let $S, R$ be linear operators on a Banach space.

(i) $R$ is called relatively bounded with respect to $S$ or $S$-bounded if $\mathcal{D}(S) \subset \mathcal{D}(R)$ and there exist $a, b \geq 0$ such that

$$
\|R x\| \leq a\|x\|+b\|S x\|, \quad x \in \mathcal{D}(S)
$$

the infimum of all $b$ such that (10) holds with some $a \geq 0$ is called the relative bound of $R$ with respect to $S$ or $S$-bound of $R$. 
(ii) $R$ is called $p$-subordinate to $S$ with $p \in[0,1]$ if $\mathcal{D}(S) \subset \mathcal{D}(R)$ and there exists $c \geq 0$ such that

$$
\|R x\| \leq c\|x\|^{1-p}\|S x\|^{p}, \quad x \in \mathcal{D}(S)
$$

the minimal constant $c$ such that (11) holds is called the $p$-subordination bound of $R$ to $S$.

Note that, in contrast to the relative bound, the infimum over all $c$ that satisfy (11) does itself satisfy (11) and hence the $p$-subordination bound is indeed a minimum.

LEMMA 3.2. Let $S, R$ be linear operators with $\mathcal{D}(S) \subset \mathcal{D}(R)$ and let $p \in[0,1]$.

(i) $R$ is $p$-subordinate to $S$ if and only if there exists a constant $c^{\prime} \geq 0$ such that

$$
\|R x\| \leq c^{\prime}\left(\varepsilon^{-p}\|x\|+\varepsilon^{1-p}\|S x\|\right), \quad x \in \mathcal{D}(S), \varepsilon>0 .
$$

(ii) If $R$ is $p$-subordinate to $S$ with $p<1$, then $R$ is $S$-bounded with $S$-bound 0 .

(iii) If $0 \in \varrho(S)$ and $R$ is $p$-subordinate to $S$, then $R$ is $q$-subordinate to $S$ for every $q>p$.

Proof. (i) was proved in [20, p. 146], (ii) follows from (i), and (iii) is a consequence of the inequality $\|x\|^{1-p} \leq\|x\|^{1-q}\left\|S^{-1}\right\|^{q-p}\|S x\|^{q-p}, x \in \mathcal{D}(S)$.

The following lemma provides conditions guaranteeing that e.g. a multiplication operator $R$ in $L^{q}(\Omega)$ with $q \in\left[1, \infty\left[\right.\right.$ and open $\Omega \subset \mathbb{R}^{n}$ is $p$-subordinate to an elliptic partial differential operator $S$ of order $m>0$; more generally, $R$ may also be a partial differential operator of order $k \leq m$.

In fact, if $W^{m, q}(\Omega)$ denotes the usual Sobolev space of $m$ times weakly differentiable functions with derivatives in $L^{q}(\Omega)$, then we consider operators $S$ on $L^{q}(\Omega)$ such that $\mathcal{D}(S) \subset W^{m, q}(\Omega)$ and $S$ satisfies a so-called a priori estimate,

$$
\|u\|_{W^{m, q}(\Omega)} \leq c_{0}\left(\|u\|_{L^{q}(\Omega)}+\|S u\|_{L^{q}(\Omega)}\right), \quad u \in \mathcal{D}(S)
$$

with some constant $c_{0}>0$; such an estimate holds e.g. if $S$ is a properly elliptic partial differential operator of order $m$ with appropriate boundary conditions, see [27, Chap. 2, §5], [36, §5.3].

LEMMA 3.3. Let $S$ be a linear operator on $L^{q}(\Omega), q \in[1, \infty[$, so that $0 \in \varrho(S)$, $\mathcal{D}(S) \subset W^{m, q}(\Omega)$ and an a priori estimate (13) holds.

(i) Let $\Omega=\mathbb{R}^{n}, g: \mathbb{R}^{n} \rightarrow \mathbb{C}$ a locally integrable function, and let $R$ be the corresponding (maximal) multiplication operator,

$$
R u:=g u, \quad \mathcal{D}(R):=\left\{u \in L^{q}\left(\mathbb{R}^{n}\right) \mid g u \in L^{q}\left(\mathbb{R}^{n}\right)\right\} .
$$

If there exist $s \in[0, n]$ and $c_{1}>0$ such that

$$
\int_{B_{r}\left(x_{0}\right)}|g(x)|^{q} \mathrm{~d} x \leq c_{1} r^{s}, \quad x_{0} \in \mathbb{R}^{n}, 0<r<1,
$$


and if

$$
\left\{\begin{array}{l}
s>n-m q \text { if } q>1 \\
s \geq n-m \text { if } q=1
\end{array}\right.
$$

then $R$ is $p$-subordinate to $S$ with $p=\frac{1}{m q}(n-s)$.

(ii) If $R$ is a partial differential operator on $L^{q}(\Omega)$ of order $k \leq m$ with coefficients in $L^{\infty}(\Omega)$, then $R$ is $\frac{k}{m}$-subordinate to $S$.

Proof. (i) Consider the measure $\mu$ on $\mathbb{R}^{n}$ given by $\mu(A)=\int_{A}|g(x)|^{q} \mathrm{~d} x$. Then $\|g u\|_{L^{q}\left(\mathbb{R}^{n}\right)}=\|u\|_{L^{q}(\mu)}$. Due to assumption (14), we can apply [29, $\S 1.4 .7$, Corollary 1] and estimate

$$
\|u\|_{L^{q}(\mu)} \leq c_{2}\|u\|_{W^{m, q}\left(\mathbb{R}^{n}\right)}^{p}\|u\|_{L^{q}\left(\mathbb{R}^{n}\right)}^{1-p}, \quad u \in W^{m, q}\left(\mathbb{R}^{n}\right),
$$

with some constant $c_{2}>0$. The estimate (13) together with $0 \in \varrho(S)$ implies that

$$
\|u\|_{W^{m, q}\left(\mathbb{R}^{n}\right)} \leq c_{0}\left(1+\left\|S^{-1}\right\|\right)\|S u\|_{L^{q}\left(\mathbb{R}^{n}\right)}, \quad u \in \mathcal{D}(S),
$$

and hence the subordination inequality (11) follows.

(ii) The proof of (ii) is similar to that of (i) if, instead of (15), we use the interpolation inequality for intermediate derivatives, see [2, Theorem 5.2],

$$
\|u\|_{W^{k, q}(\Omega)} \leq c\|u\|_{W^{m, q}(\Omega)}^{k / m}\|u\|_{L^{q}(\Omega)}^{1-k / m}, \quad u \in W^{k, q}(\Omega) .
$$

REMARK 3.4. The subordination property in (ii) was used e.g. in $[28, \S 10]$ and $[40,41]$ to obtain expansions in eigenfunctions of $S+R$.

Next we show that bisectoriality is stable under $p$-subordinate perturbations and we study their effect on the spectrum, see Fig. 2.

LEMMA 3.5. Let $S, R$ be linear operators, $S$ bisectorial with angle $\theta \in[0, \pi / 2[$ and radius $r \geq 0$, and $R p$-subordinate to $S$ with $p \in[0,1]$.

(i) For every $\left.\left.\theta^{\prime} \in\right] \theta, \pi / 2\right]$ there exists $M^{\prime} \geq 0$ such that

$$
\left\|R(S-z)^{-1}\right\| \leq \frac{M^{\prime}}{|z|^{1-p}}, \quad z \in \Omega_{\theta^{\prime}, r},
$$

where $\Omega_{\theta^{\prime}, r}=\left\{z \in \mathbb{C}\left|\theta^{\prime} \leq\right| \arg z\left|\leq \pi-\theta^{\prime},\right| z \mid>r\right\}$, see (7).

(ii) If $R$ is even $p$-subordinate to $S$ with $p<1$, then for every $\left.\theta^{\prime} \in\right] \theta, \pi / 2[$ there exists $r^{\prime} \geq r$ such that $S+R$ is bisectorial with angle $\theta^{\prime}$ and radius $r^{\prime}$.

Proof. (i) Let $\left.\left.\theta^{\prime} \in\right] \theta, \pi / 2\right]$. Then for $z \in \Omega_{\theta^{\prime}, r}$, we use (6) to estimate

$$
\left\|S(S-z)^{-1}\right\| \leq 1+|z| \cdot\left\|(S-z)^{-1}\right\| \leq 1+M .
$$



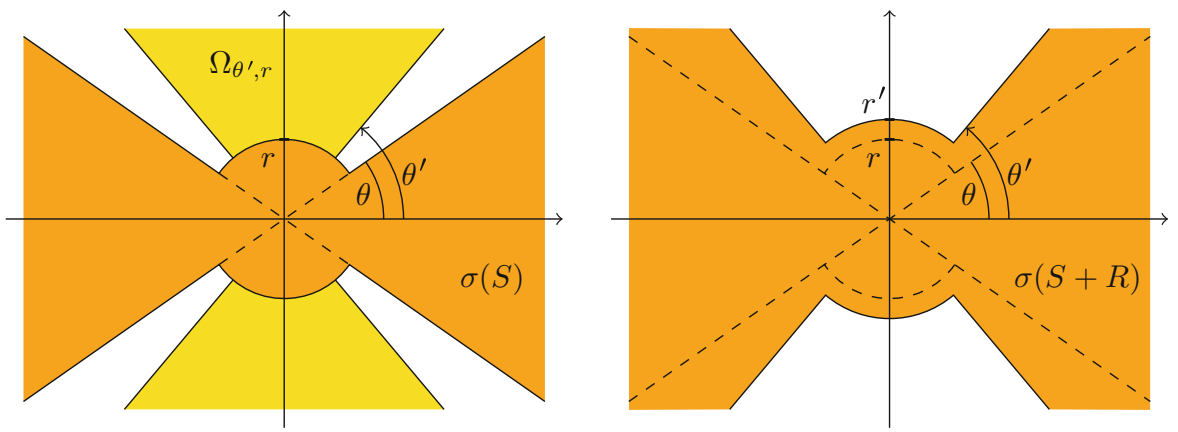

Figure 2. The perturbation of the spectrum of a bisectorial operator in Lemma 3.5

Hence if $R$ is $p$-subordinate to $S$, then

$$
\left\|R(S-z)^{-1}\right\| \leq c\left\|(S-z)^{-1}\right\|^{1-p}\left\|S(S-z)^{-1}\right\|^{p} \leq c \cdot\left(\frac{M}{|z|}\right)^{1-p}(1+M)^{p} .
$$

(ii) Let $\left.\theta^{\prime} \in\right] \theta, \pi / 2\left[\right.$. By (16) there exists $r^{\prime} \geq r$ such that $\left\|R(S-z)^{-1}\right\| \leq 1 / 2$ for all $z \in \Omega_{\theta^{\prime}, r^{\prime}}$. A Neumann series argument then implies that $z \in \varrho(S+R)$ for $z \in \Omega_{\theta^{\prime}, r^{\prime}}$,

$$
(S+R-z)^{-1}=(S-z)^{-1}\left(I+R(S-z)^{-1}\right)^{-1},
$$

and $\left\|(S+R-z)^{-1}\right\| \leq 2\left\|(S-z)^{-1}\right\|$. This and (6) imply that $S+R$ is bisectorial with angle $\theta^{\prime}$ and radius $r^{\prime}$.

REMARK 3.6. If the unperturbed operator $S$ in Lemma 3.5 is selfadjoint, and hence bisectorial with angle $\theta=0$, then the spectral inclusion implied by Lemma 3.5 (ii) and displayed in Fig. 2 follows from the spectral enclosure [12, Corollary 2.4] since $p$-subordinate perturbations with $p<1$ have relative bound 0 .

For bisectorial operators with radius $r=0$, the estimate (16) is, in fact, an equivalent characterisation of $p$-subordinacy:

LEMMA 3.7. Let $S, R$ be linear operators, $S$ bisectorial with angle $\theta \in[0, \pi / 2[$ and radius $r \geq 0, \mathcal{D}(S) \subset \mathcal{D}(R)$, and $p \in[0,1]$. If there exist $\left.\left.\theta^{\prime} \in\right] \theta, \pi / 2\right]$ and $M^{\prime} \geq 0$ with

$$
\left\|R(S-z)^{-1}\right\| \leq \frac{M^{\prime}}{|z|^{1-p}}, \quad z \in \Omega_{\theta^{\prime}, 0},
$$

where $\Omega_{\theta^{\prime}, 0}=\left\{z \in \mathbb{C}\left|\theta^{\prime} \leq\right| \arg z\left|\leq \pi-\theta^{\prime},\right| z \mid>0\right\}$, then $R$ is p-subordinate to $S$. Proof. The estimate (17) implies that

$$
\|R x\| \leq \frac{M^{\prime}}{|z|^{1-p}}\|(S-z) x\| \leq M^{\prime}\left(|z|^{p}\|x\|+|z|^{p-1}\|S x\|\right), \quad x \in \mathcal{D}(S) .
$$

Choosing $z=\mathrm{i} \varepsilon^{-1}, \varepsilon>0$, we obtain (12); Lemma 3.2 (i) thus yields the claim. 
REMARK 3.8. A result analogous to Lemma 3.5 holds for any subset $\Omega \subset$ $\varrho(S) \backslash\{0\}$ such that there is an estimate (6) on $\Omega$ instead of $\Omega_{\theta^{\prime}, r}$; in this case, $S$ is not required to be bisectorial. In the same way Lemma 3.7 can be generalised if, in addition, $\Omega$ satisfies the condition $\{|z| \mid z \in \Omega\}=\mathbb{R}_{+}$.

The following theorem on $p$-subordinate perturbations of dichotomous bisectorial operators is crucial for the next sections. Compared to [25, Theorem 1.3] we use $p$-subordinacy rather than an estimate of type (16) and we only assume that the imaginary axis belongs to the set of points of regular type of the perturbed operator, not to its resolvent set.

Recall that for a linear operator $S$ on a Banach space, $z \in \mathbb{C}$ is called a point of regular type if there exists $c>0$ such that

$$
\|(S-z) x\| \geq c\|x\|, \quad x \in \mathcal{D}(S) .
$$

The set $r(S)$ of all points of regular type is open and satisfies $\varrho(S) \subset r(S)$. If $\Omega \subset r(T)$ is a connected subset such that $\Omega \cap \varrho(S) \neq \varnothing$, then $\Omega \subset \varrho(S)$, see [3, §78]. The complement $\mathbb{C} \backslash r(S)$ is the approximate point spectrum of $S$.

THEOREM 3.9. Let $S$ be a closed densely defined linear operator on a Banach space $V$ such that

(i) $i \mathbb{R} \subset \varrho(S)$;

(ii) $S$ is bisectorial with angle $\theta \in[0, \pi / 2$ [ and radius $r \geq 0$;

(iii) the integral $\int_{\mathrm{i} \mathbb{R}}^{\prime}(S-z)^{-1} x \mathrm{~d} z$ exists for all $x \in V$.

Let $R$ be p-subordinate to $S$ with $p<1$. If $\mathrm{i} \mathbb{R} \subset r(S+R)$, then $S+R$ is dichotomous with dichotomy gap $h>0$, the corresponding projections $P_{ \pm}$satisfy

$$
\frac{1}{\pi \mathrm{i}} \int_{\mathrm{i} \mathbb{R}}^{\prime}(S+R-z)^{-1} x \mathrm{~d} z=P_{+} x-P_{-} x, \quad x \in V,
$$

and $S+R$ is bisectorial with some angle $\left.\theta^{\prime \prime} \in\right] \theta, \pi / 2\left[\right.$. Moreover, for every $\theta^{\prime} \in$ ]$\theta, \pi / 2\left[\right.$ there exists $r^{\prime} \geq r$ such that $S+R$ is also bisectorial with angle $\theta^{\prime}$ and radius $r^{\prime}$ and (see Fig. 3)

$$
\{z \in \mathbb{C}|| \operatorname{Re} z \mid<h\} \cup \Omega_{\theta^{\prime \prime}, 0} \cup \Omega_{\theta^{\prime}, r^{\prime}} \subset \varrho(S+R) .
$$

Proof. Lemma 3.5 implies the bisectoriality with angle $\theta^{\prime}$ and radius $r^{\prime}$. In particular, the connected subset $\mathrm{i} \mathbb{R}$ of $r(S+R)$ contains points from $\varrho(S+R)$ and thus $\mathrm{i} \mathbb{R} \subset$ $\varrho(S+R)$. Since $\varrho(S+R)$ is open and $(S+R-z)^{-1}$ is uniformly bounded on compact subsets, there exist $\left.h>0, \theta^{\prime \prime} \in\right] \theta, \pi / 2[$ such that $S+R$ is bisectorial with angle $\theta^{\prime \prime}$ and (19) holds. Consequently, $S+R$ satisfies the assumptions (i) and (ii) in Theorem 2.6. Furthermore, (16) and the estimate (6) for $S+R$ imply that

$$
\int_{\mathrm{i} \mathbb{R}}(S+R-z)^{-1} R(S-z)^{-1} \mathrm{~d} z
$$




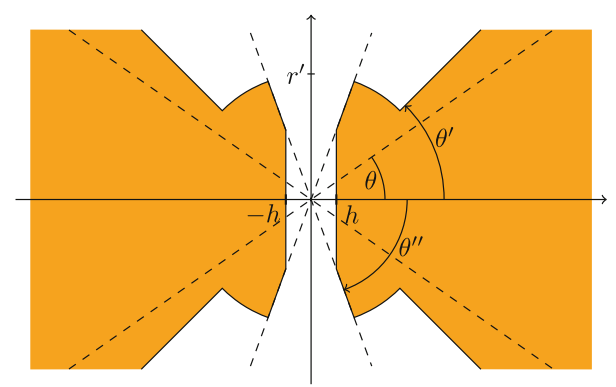

Figure 3. Region containing $\sigma(S+R)$ in Theorem 3.9

exists in the uniform operator topology. From the resolvent identity

$$
(S+R-z)^{-1}=(S-z)^{-1}-(S+R-z)^{-1} R(S-z)^{-1}, \quad z \in \mathrm{i} \mathbb{R},
$$

we conclude that $S+R$ also satisfies assumption (iii) in Theorem 2.6.

In view of Lemma 2.12 , the previous result immediately applies to sectorially dichotomous operators.

COROLLARY 3.10. Let $S$ be sectorially dichotomous with angle $\theta \in[0, \pi / 2[$. Let $R$ be $p$-subordinate to $S$ with $p<1$ and $\mathrm{i} \mathbb{R} \subset r(S+R)$. Then $S+R$ is dichotomous and all assertions of Theorem 3.9 hold.

\section{Dichotomous Hamiltonians}

Hamiltonian operator matrices are block operator matrices of a particular form. Block operator matrices can be classified according to the domains of their entries into diagonally dominant, off-diagonally dominant, and top dominant, see [33-35]. Here we introduce the new class of diagonally $p$-dominant block operator matrices.

DEFINITION 4.1. Let $H_{1}, H_{2}$ be Hilbert spaces, consider densely defined linear operators $A$ in $H_{1}, B$ from $H_{2}$ to $H_{1}, C$ from $H_{1}$ to $H_{2}$, and $D$ in $H_{2}$, and let $p \in[0,1]$. Then the block operator matrix

$$
T=\left(\begin{array}{ll}
A & B \\
C & D
\end{array}\right) \quad \text { in } H_{1} \times H_{2}
$$

is called

(i) diagonally dominant if $C$ is $A$-bounded and $B$ is $D$-bounded;

(ii) diagonally $p$-dominant if $C$ is $p$-subordinate to $A$ and $B$ is $p$-subordinate to $D$.

Note that for a diagonally dominant block operator matrix, the domain of $T$ is given by the domains of the two diagonal entries, $\mathcal{D}(T)=\mathcal{D}(A) \times \mathcal{D}(D)$. By Lemma 3.2 (ii), every diagonally $p$-dominant block operator matrix is diagonally dominant. 
If we decompose a block operator matrix $T$ into its diagonal and off-diagonal part,

$$
T=S+R \quad \text { with } S:=\left(\begin{array}{cc}
A & 0 \\
0 & D
\end{array}\right), \quad R:=\left(\begin{array}{cc}
0 & B \\
C & 0
\end{array}\right),
$$

then $T$ is diagonally dominant if and only if $R$ is $S$-bounded, see [34, §2.2]. A similar statement holds for diagonal $p$-dominance:

LEMMA 4.2. (i) A block operator matrix $T$ is diagonally $p$-dominant if and only if $R$ is $p$-subordinate to $S$.

(ii) If $0 \in \varrho(A) \cap \varrho(D), C$ is $p_{1}$-subordinate to $A$, and $B$ is $p_{2}$-subordinate to $D$, then $T$ is diagonally $p$-dominant with $p=\max \left\{p_{1}, p_{2}\right\}$.

Proof. (i) If $T$ is diagonally $p$-dominant, then Hölder's inequality yields that, for $x=(u, v) \in \mathcal{D}(T)=\mathcal{D}(S)$,

$$
\begin{aligned}
\|R x\|^{2} & =\|B v\|^{2}+\|C u\|^{2} \leq c_{B}^{2}\|v\|^{2(1-p)}\|D v\|^{2 p}+c_{C}^{2}\|u\|^{2(1-p)}\|A u\|^{2 p} \\
& \leq \max \left\{c_{B}^{2}, c_{C}^{2}\right\}\left(\|u\|^{2}+\|v\|^{2}\right)^{1-p}\left(\|A u\|^{2}+\|D v\|^{2}\right)^{p} \\
& =\max \left\{c_{B}^{2}, c_{C}^{2}\right\}\|x\|^{2(1-p)}\|S x\|^{2 p} .
\end{aligned}
$$

Hence $R$ is $p$-subordinate to $S$. Vice versa, let $R$ be $p$-subordinate to $S$. Then for $u \in \mathcal{D}(A)$, we have $x:=(u, 0) \in \mathcal{D}(S) \subset \mathcal{D}(R)$, i.e. $u \in \mathcal{D}(C)$, and

$$
\|C u\|=\|R x\| \leq c\|x\|^{1-p}\|S x\|^{p}=c\|u\|^{1-p}\|A u\|^{p} .
$$

An analogous argument yields that $B$ is $p$-subordinate to $D$.

(ii) is an immediate consequence of Lemma 3.2 (iii).

DEFINITION 4.3. A block operator matrix $T$ is called Hamiltonian if $H_{1}=H_{2}=$ $H$ and $T$ has the form

$$
T=\left(\begin{array}{cc}
A & B \\
C & -A^{*}
\end{array}\right) \quad \text { in } H \times H
$$

with $A$ closed and $B, C$ symmetric; $T$ is called nonnegative if $B, C$ are nonnegative.

REMARK 4.4. A Hamiltonian $T$ is diagonally dominant if and only if $\mathcal{D}(A) \subset$ $\mathcal{D}(C)$ and $\mathcal{D}\left(A^{*}\right) \subset \mathcal{D}(B)$, see [34, Remark 2.2.2].

LEMMA 4.5. Let $T$ be a nonnegative diagonally dominant Hamiltonian operator matrix such that $\mathrm{i} \mathbb{R} \subset \varrho(A)$. Then $\mathrm{i} \mathbb{R} \subset r(T)$.

Proof. Since $B, C$ are nonnegative symmetric, they admit nonnegative selfadjoint extensions. We may thus assume that $B$ and $C$ are selfadjoint. Then for $t \in \mathbb{R}$, the operator $C^{1 / 2}(A-\mathrm{i} t)^{-1}$ is defined on $H$ and closed; hence it is bounded by the closed graph theorem. Analogously, $B^{1 / 2}\left(A^{*}+\mathrm{i} t\right)^{-1}$ is bounded. Suppose that it $\notin r(T)$. Then there exist $\left(u_{n}, v_{n}\right) \in \mathcal{D}(T)$ such that

$$
\left\|\left(\begin{array}{l}
u_{n} \\
v_{n}
\end{array}\right)\right\|=1, n \in \mathbb{N}, \quad \lim _{n \rightarrow \infty}(T-\mathrm{i} t)\left(\begin{array}{l}
u_{n} \\
v_{n}
\end{array}\right)=\lim _{n \rightarrow \infty}\left(\begin{array}{c}
(A-\mathrm{i} t) u_{n}+B v_{n} \\
C u_{n}-\left(A^{*}+\mathrm{i} t\right) v_{n}
\end{array}\right)=0 .
$$


In view of $\left\|u_{n}\right\| \leq 1,\left\|v_{n}\right\| \leq 1, n \in \mathbb{N}$, the latter implies that

$$
\begin{aligned}
& \left((A-\mathrm{i} t) u_{n} \mid v_{n}\right)+\left(B v_{n} \mid v_{n}\right) \rightarrow 0, \\
& \left(C u_{n} \mid u_{n}\right)-\left(\left(A^{*}+\mathrm{i} t\right) v_{n} \mid u_{n}\right) \rightarrow 0,
\end{aligned}
$$

Adding these two relations and taking the real part, we arrive at

$$
\left(C u_{n} \mid u_{n}\right)+\left(B v_{n} \mid v_{n}\right) \rightarrow 0, \quad n \rightarrow \infty
$$

Since $B, C$ are nonnegative, we obtain

$$
\left\|C^{1 / 2} u_{n}\right\|^{2}=\left(C u_{n} \mid u_{n}\right) \rightarrow 0, \quad\left\|B^{1 / 2} v_{n}\right\|^{2}=\left(B v_{n} \mid v_{n}\right) \rightarrow 0, \quad n \rightarrow \infty .
$$

Because of $\left\|v_{n}\right\| \leq 1, n \in \mathbb{N}$, the sequences $\left((A-\mathrm{i} t)^{-1} v_{n}\right)_{n}$ and $\left(C^{1 / 2}(A-\mathrm{i} t)^{-1} v_{n}\right)_{n}$ are bounded, and thus

$$
\begin{aligned}
0 & =\lim _{n \rightarrow \infty}\left(C u_{n}-\left(A^{*}+\mathrm{i} t\right) v_{n} \mid(A-\mathrm{i} t)^{-1} v_{n}\right) \\
& =\lim _{n \rightarrow \infty}\left(\left(C^{1 / 2} u_{n} \mid C^{1 / 2}(A-\mathrm{i} t)^{-1} v_{n}\right)-\left\|v_{n}\right\|^{2}\right)=-\lim _{n \rightarrow \infty}\left\|v_{n}\right\|^{2},
\end{aligned}
$$

i.e. $v_{n} \rightarrow 0, n \rightarrow \infty$. Analogous considerations for $\left((A-\mathrm{i} t) u_{n}+B v_{n} \mid\left(A^{*}+\mathrm{i} t\right)^{-1} u_{n}\right)$ yield that $u_{n} \rightarrow 0, n \rightarrow \infty$, a contradiction to $\left\|\left(u_{n}, v_{n}\right)\right\|=1, n \in \mathbb{N}$.

The following theorem is a perturbation result for Hamiltonians $T$ with sectorially dichotomous $A$; the corresponding spectral enclosure is displayed in Fig. 3.

THEOREM 4.6. Let $T$ be a nonnegative diagonally p-dominant Hamiltonian with $p<1$ and let $A$ be sectorially dichotomous with angle $\theta \in[0, \pi / 2[$. Then, $T$ is dichotomous, the spectral projections $P_{+}, P_{-}$satisfy

$$
\frac{1}{\pi \mathrm{i}} \int_{\mathrm{i} \mathbb{R}}^{\prime}(T-z)^{-1} x \mathrm{~d} z=P_{+} x-P_{-} x, \quad x \in H \times H,
$$

and there exist $\left.h>0, \theta^{\prime \prime} \in\right] \theta, \pi / 2\left[\right.$ and for every $\left.\theta^{\prime} \in\right] \theta, \pi / 2\left[\right.$ an $r^{\prime}>0$ such that

$$
\{z \in \mathbb{C}|| \operatorname{Re} z \mid<h\} \cup \Omega_{\theta^{\prime \prime}, 0} \cup \Omega_{\theta^{\prime}, r^{\prime}} \subset \varrho(T) .
$$

Proof. We consider the decomposition $T=S+R$ into diagonal and off-diagonal part,

$$
T=S+R \quad \text { with } S:=\left(\begin{array}{cc}
A & 0 \\
0 & -A^{*}
\end{array}\right), \quad R:=\left(\begin{array}{cc}
0 & B \\
C & 0
\end{array}\right) .
$$

Since $A$ and hence $A^{*}$ are sectorially dichotomous, see Corollary 2.16 , the same is true for $S$. Moreover, $R$ is $p$-subordinate to $S$ and $i \mathbb{R} \subset r(T)$. Thus Corollary 3.10 applies and yields all claims.

A Hamiltonian $T$ as in (21) does not have any symmetry properties with respect to the scalar product in the Hilbert space $H \times H$. However, it exhibits some symmetries with respect to two different indefinite inner products on $H \times H$, see [24, Section 5]. 
A vector space $V$ together with an indefinite inner product $[\cdot \mid \cdot]$ is called a Krein space if there exists a scalar product $(\cdot \mid \cdot)$ on $V$ and an involution $J: V \rightarrow V$ such that $(V,(\cdot \mid \cdot))$ is a Hilbert space and

$$
[x \mid y]=(J x \mid y), \quad x, y \in V .
$$

The so-called fundamental symmetry $J$ and the scalar product are not unique, but the norms induced by two such scalar products are equivalent.

A subspace $U \subset V$ is called $J$-nonnegative ( $J$-nonpositive, $J$-neutral, respectively, ) if $[x \mid x] \geq 0$ ( $\leq 0,=0$, respectively) for all $x \in U$. A subspace $U$ is $J$-neutral if and only if it is contained in its $J$-orthogonal complement $U^{\langle\perp\rangle}$,

$$
U \subset U^{\langle\perp\rangle}:=\{x \in V \mid[x \mid y]=0 \text { for all } y \in U\},
$$

and it is called hypermaximal $J$-neutral if $U=U^{\langle\perp\rangle}$.

A linear operator $T$ on $V$ is called $J$-accretive if $\operatorname{Re}[T x \mid x] \geq 0$ for all $x \in \mathcal{D}(T)$. A densely defined linear operator $T$ is called $J$-skew-symmetric if $[T x \mid y]=-[x \mid T y]$ for all $x, y \in \mathcal{D}(T)$. For more results on Krein spaces and operators therein, we refer to $[7,19]$.

PROPOSITION 4.7. Let $V$ be a Krein space with fundamental symmetry $J$ and let $T$ be a dichotomous operator on $V$ with corresponding decomposition $V=V_{+} \oplus V_{-}$ and projections $P_{+}, P_{-}$such that

$$
\frac{1}{\pi \mathrm{i}} \int_{\mathrm{i} \mathbb{R}}^{\prime}(T-z)^{-1} x \mathrm{~d} z=P_{+} x-P_{-} x, \quad x \in V .
$$

(i) If $T$ is $J$-accretive, then $V_{+}$is $J$-nonnegative and $V_{-}$is $J$-nonpositive.

(ii) If $T$ is $J$-skew-symmetric, then $V_{+}$and $V_{-}$are hypermaximal $J$-neutral.

Proof. (i) The simple proof was given in [25, Theorem 1.4]; e.g. for $x \in V_{+}$it is nothing but the inequality

$$
\begin{aligned}
{[x \mid x] } & =\operatorname{Re}\left[P_{+} x-P_{-} x \mid x\right]=\frac{1}{\pi} \int_{\mathbb{R}}^{\prime} \operatorname{Re}\left[(T-\mathrm{i} t)^{-1} x \mid x\right] \mathrm{d} t \\
& =\frac{1}{\pi} \int_{\mathbb{R}}^{\prime} \operatorname{Re}\left[T(T-\mathrm{i} t)^{-1} x \mid(T-\mathrm{i} t)^{-1} x\right] \mathrm{d} t \geq 0 .
\end{aligned}
$$

(ii) If $T$ is $J$-skew-symmetric, then both $T$ and $-T$ are $J$-accretive. Consequently, $V_{ \pm}$are both nonnegative and nonpositive, thus neutral. To prove hypermaximal neutrality, let e.g. $x \in V_{+}^{\langle\perp\rangle}$. Using the decomposition $V=V_{+} \oplus V_{-}$, we write $x=u+v$ with $u \in V_{+}, v \in V_{-}$. If $v \neq 0$, then there exists $y \in V$ such that $[v \mid y] \neq 0$ (e.g. one may choose $y=J v$ ). Since $V_{-}$is neutral, we may assume that $y \in V_{+}$. The neutrality of $V_{+}$then implies that $[x \mid y]=[v \mid y] \neq 0$, in contradiction to $x \in V_{+}^{\langle\perp\rangle}$. Therefore $v=0$, i.e. $x \in V_{+}$. 
Following [24, Section 5], we equip the product space $H \times H$ with two different indefinite inner products, given by $[x \mid y]_{1}:=\left(J_{1} x \mid y\right)$ and $[x \mid y]_{2}:=\left(J_{2} x \mid y\right)$ with the fundamental symmetries

$$
J_{1}:=\left(\begin{array}{cc}
0 & -\mathrm{i} I \\
\mathrm{i} I & 0
\end{array}\right), \quad J_{2}:=\left(\begin{array}{ll}
0 & I \\
I & 0
\end{array}\right)
$$

As in the case of bounded $B$ and $C$, the Hamiltonian has the following symmetry properties with respect to $J_{1}$ and $J_{2}$.

LEMMA 4.8. The Hamiltonian operator matrix $T$ is $J_{1}$-skew-symmetric, and $T$ is nonnegative if and only if it is $J_{2}$-accretive.

Proof. The assertions are immediate from

$$
\begin{aligned}
{[T(u, v) \mid(u, v)]_{1} } & =\mathrm{i}(2 \operatorname{Re}(A u \mid v)+(B v \mid v)-(C u \mid u)) \in \mathrm{i} \mathbb{R}, \\
\operatorname{Re}[T(u, v) \mid(u, v)]_{2} & =(B v \mid v)+(C u \mid u) .
\end{aligned}
$$

COROLLARY 4.9. In the situation of Theorem 4.6, let $H \times H=V_{+} \oplus V_{-}$be the decomposition corresponding to the dichotomy of $T$. Then $V_{+}, V_{-}$are hypermaximal $J_{1}$-neutral, $V_{+}$is $J_{2}$-nonnegative, and $V_{-}$is $J_{2}$-nonpositive.

\section{Invariant graph subspaces and Riccati equations}

There is a close relation between the invariance of graph subspaces

$$
G(X):=\left\{\left(\begin{array}{c}
u \\
X u
\end{array}\right) \mid u \in \mathcal{D}(X)\right\}
$$

of linear operators $X$ on a Hilbert space $H$ under a block operator matrix and solutions of Riccati equations, see e.g. $[8,18,41]$; in our setting it reads as follows:

LEMMA 5.1. Let $T$ be a diagonally dominant Hamiltonian and $X$ a linear operator on $H$. Then the graph subspace $G(X)$ is $T$-invariant if and only if $X$ is a solution of the Riccati equation

$$
\left(A^{*} X+X(A+B X)-C\right) u=0, \quad u \in \mathcal{D}(A) \cap X^{-1} \mathcal{D}\left(A^{*}\right)
$$

in particular, $(A+B X) u \in \mathcal{D}(X)$ for all $u \in \mathcal{D}(A) \cap X^{-1} \mathcal{D}\left(A^{*}\right)$.

Proof. $G(X)$ is $T$-invariant if and only if for all $u \in \mathcal{D}(A) \cap \mathcal{D}(X)$ with $X u \in \mathcal{D}\left(A^{*}\right)$ there exists $v \in \mathcal{D}(X)$ such that

$$
\left(\begin{array}{c}
A u+B X u \\
C u-A^{*} X u
\end{array}\right)=T\left(\begin{array}{c}
u \\
X u
\end{array}\right)=\left(\begin{array}{c}
v \\
X v
\end{array}\right),
$$

and this is obviously equivalent to (24). 
Graph subspaces are closely related to the Krein space fundamental symmetries $J_{1}, J_{2}$ introduced in (23), see also [14]:

LEMMA 5.2. ([41, Lemma 6.2]) Let $X$ be a linear operator on $H$. Then

(i) $X$ is selfadjoint if and only if $G(X)$ is hypermaximal $J_{1}$-neutral;

if $X$ is symmetric, then

(ii) $X$ is nonnegative (nonpositive, respectively) if and only if $G(X)$ is $J_{2}$-nonnegative ( $J_{2}$-nonpositive, respectively).

The next theorem generalises [24, Theorem 5.1] where the off-diagonal operators $B$ and $C$ were assumed to be bounded, and it complements results in $[21,41,42]$ where Hamiltonians $T$ possessing a Riesz basis of generalised eigenvectors but without dichotomy were investigated.

THEOREM 5.3. Let $T$ be a nonnegative diagonally p-dominant Hamiltonian with $p<1$ such that $A$ is sectorially dichotomous and

$$
\bigcap_{t \in \mathbb{R}} \operatorname{ker}\left(B\left(A^{*}+\mathrm{i} t\right)^{-1}\right)=\{0\} .
$$

Then

(i) $T$ is dichotomous and its spectral subspaces are graph subspaces, $V_{ \pm}=G\left(X_{ \pm}\right)$;

(ii) $X_{ \pm}$are selfadjoint, $X_{+}$is nonnegative, and $X_{-}$is nonpositive;

(iii) $\mathcal{D}(A) \cap X_{ \pm}^{-1} \mathcal{D}\left(A^{*}\right)$ are a core for $X_{ \pm}$and $X_{ \pm}$satisfy the Riccati equations

$$
\left(A^{*} X_{ \pm}+X_{ \pm}\left(A+B X_{ \pm}\right)-C\right) u=0, \quad u \in \mathcal{D}(A) \cap X_{ \pm}^{-1} \mathcal{D}\left(A^{*}\right) .
$$

Proof. (i) By Theorem 4.6 and Corollary 4.9, $T$ is dichotomous, $V_{+}, V_{-}$are hypermaximal $J_{1}$-neutral, $V_{+}$is $J_{2}$-nonnegative, and $V_{-}$is $J_{2}$-nonpositive. To show that $V_{ \pm}=G\left(X_{ \pm}\right)$with some linear operator $X_{ \pm}$, it suffices to show that $(0, w) \in V_{ \pm}$ implies $w=0$. Setting $(u, v):=(T-\mathrm{i} t)^{-1}(0, w)$ for $t \in \mathbb{R}$, we have

$$
(A-\mathrm{i} t) u+B v=0, \quad C u-\left(A^{*}+\mathrm{i} t\right) v=w .
$$

Since $V_{ \pm}$is $J_{1}$-neutral and invariant under $(T-\mathrm{i} t)^{-1}$, this implies that

$$
0=\left[\left(\begin{array}{l}
0 \\
w
\end{array}\right) \mid\left(\begin{array}{l}
u \\
v
\end{array}\right)\right]_{1}=-\mathrm{i}(w \mid u)
$$

and thus

$$
0=(w \mid u)=(C u \mid u)-(v \mid(A-\mathrm{i} t) u)=(C u \mid u)+(B v \mid v) .
$$

Since $B$ and $C$ are nonnegative, it follows that $0=(C u \mid u)=(B v \mid v)$. Thus for all $r \in \mathbb{R}$ and $\tilde{v} \in \mathcal{D}(B)$,

$$
0 \leq(B(r v+\tilde{v}) \mid r v+\tilde{v})=2 r \operatorname{Re}(B v \mid \tilde{v})+(B \tilde{v} \mid \tilde{v}),
$$


which yields $B v=0$. Similarly, we obtain $C u=0$ and so $w=-\left(A^{*}+\mathrm{i} t\right) v$. We conclude that $B\left(A^{*}+\mathrm{i} t\right)^{-1} w=-B v=0$. As $t \in \mathbb{R}$ was arbitrary, (25) implies that $w=0$.

(ii), (iii) Since $V_{ \pm}=G\left(X_{ \pm}\right)$are hypermaximal $J_{1}$-neutral and $J_{2}$-nonnegative/ nonpositive, Lemma 5.2 shows that $X_{ \pm}$are selfadjoint and nonnegative/nonpositive, while Lemma 5.1 shows that $X_{ \pm}$satisfy (26). Moreover, we have $\left(u, X_{ \pm} u\right) \in \mathcal{D}(T)$ if and only if $u \in \mathcal{D}(A) \cap X_{ \pm}^{-1} \mathcal{D}\left(A^{*}\right)$. Since $V_{ \pm} \cap \mathcal{D}(T)$ are dense in $V_{ \pm}$, this implies that $\mathcal{D}(A) \cap X_{ \pm}^{-1} \mathcal{D}\left(A^{*}\right)$ are a core for $X_{ \pm}$.

Next we derive necessary as well as sufficient conditions for assumption (25).

PROPOSITION 5.4. Let $A$ be a closed densely defined linear operator with $\mathrm{i} \mathbb{R} \subset$ $\varrho(A)$ and $B$ symmetric with $\mathcal{D}\left(A^{*}\right) \subset \mathcal{D}(B)$. Then the assertions

(i) $\operatorname{ker} B=\{0\}$,

(ii) $\bigcap_{t \in \mathbb{R}} \operatorname{ker}\left(B\left(A^{*}+\mathrm{i} t\right)^{-1}\right)=\{0\}$, see $(25)$,

(iii) $\overline{\operatorname{span}\left\{(A-\mathrm{i} t)^{-1} B^{*} u \mid t \in \mathbb{R}, u \in \mathcal{D}\left(B^{*}\right)\right\}}=H$,

(iv) $\forall \lambda \in \sigma_{p}\left(A^{*}\right): \operatorname{ker} B \cap \operatorname{ker}\left(A^{*}-\lambda\right)=\{0\}$

satisfy the implications

$$
\text { (i) } \Longrightarrow \text { (ii) } \Longleftrightarrow \text { (iii) } \Longrightarrow \text { (iv); }
$$

if A has compact resolvent and possesses a complete system of generalised eigenvectors, then even

$$
\text { (iii) } \Longleftrightarrow \text { (iv). }
$$

REMARK 5.5. For the special case of bounded $B$, instead of (25), the equivalent condition (iii) in Proposition 5.4 was used in [24, Theorem 5.1]. For the special case of normal $A$ with compact resolvent, the equivalence (iii) $\Leftrightarrow$ (iv) in Proposition 5.4 was established in [41, Proposition 6.6].

For the proof of Proposition 5.4, we need the following lemma.

LEMMA 5.6. Let $A$ be a closed densely defined linear operator with $i \mathbb{R} \subset \varrho(A)$ and $B$ symmetric with $\mathcal{D}\left(A^{*}\right) \subset \mathcal{D}(B)$. Let $\rho_{0}$ be the connected component of $\varrho(A)$ containing $\mathrm{i} \mathbb{R}$. If $\rho \subset \rho_{0}$ has an accumulation point in $\rho_{0}$, then

$$
\begin{aligned}
\bigcap_{t \in \mathbb{R}} \operatorname{ker}\left(B\left(A^{*}+\mathrm{i} t\right)^{-1}\right) & =\bigcap_{z \in \rho} \operatorname{ker}\left(B\left(A^{*}-\bar{z}\right)^{-1}\right) \\
& =\left(\operatorname{span}\left\{(A-z)^{-1} B^{*} u \mid z \in \rho, u \in \mathcal{D}\left(B^{*}\right)\right\}\right)^{\perp} .
\end{aligned}
$$

Proof. The second identity is immediate from the identities

$$
\operatorname{ker}\left(B\left(A^{*}-\bar{z}\right)^{-1}\right)=\mathcal{R}\left(\left(B\left(A^{*}-\bar{z}\right)^{-1}\right)^{*}\right)^{\perp}=\mathcal{R}\left((A-z)^{-1} B^{*}\right)^{\perp} ;
$$


note that we have used that $B\left(A^{*}-\bar{z}\right)^{-1}$ is bounded and that $\left.\left(B\left(A^{*}-\bar{z}\right)^{-1}\right)^{*}\right|_{\mathcal{D}\left(B^{*}\right)}=$ $(A-z)^{-1} B^{*}$. Moreover, by the identity theorem, if $\left((A-z)^{-1} B^{*} u \mid x\right)=0$ for all $z \in \rho$, then this continues to hold for all $z \in \rho_{0}$ and thus

$$
\bigcap_{z \in \rho} \operatorname{ker}\left(B\left(A^{*}-\bar{z}\right)^{-1}\right)=\bigcap_{z \in \rho_{0}} \operatorname{ker}\left(B\left(A^{*}-\bar{z}\right)^{-1}\right) .
$$

Since $i \mathbb{R}$ is one possible choice for $\rho$, the proof is complete.

Proof of Proposition 5.4. The implication (i) $\Rightarrow$ (ii) is clear and (ii) $\Leftrightarrow$ (iii) follows from Lemma 5.6. For the implication (ii) $\Rightarrow$ (iv), we observe that if $\lambda \in \sigma_{p}\left(A^{*}\right)$ and $x \in \operatorname{ker} B \cap \operatorname{ker}\left(A^{*}-\lambda\right)$, then $B\left(A^{*}+\mathrm{i} t\right)^{-1} x=(\lambda+\mathrm{i} t)^{-1} B x=0$ for all $t \in \mathbb{R}$ and hence $x=0$ by (ii).

To show the reverse implication (iv) $\Rightarrow$ (ii) under the additional assumptions on $A$, we first prove that the closed subspace

$$
\mathcal{N}:=\bigcap_{t \in \mathbb{R}} \operatorname{ker}\left(B\left(A^{*}+\mathrm{i} t\right)^{-1}\right)
$$

is $\left(A^{*}-z\right)^{-1}$-invariant for every $z \in \varrho\left(A^{*}\right)$. Let $x \in \mathcal{N}$. Since $A$ has compact resolvent, $\varrho(A)$ is connected. Thus Lemma 5.6 implies that $B\left(A^{*}-z\right)^{-1} x=0$ for all $z \in \varrho\left(A^{*}\right)$. Hence by the resolvent identity, we find that for all $t \in \mathbb{R}, z \neq-\mathrm{i} t$,

$$
B\left(A^{*}+\mathrm{i} t\right)^{-1}\left(A^{*}-z\right)^{-1} x=\frac{1}{\mathrm{i} t+z}\left(B\left(A^{*}-z\right)^{-1} x-B\left(A^{*}+\mathrm{i} t\right)^{-1} x\right)=0 .
$$

Therefore $\mathcal{N}$ is $\left(A^{*}-z\right)^{-1}$-invariant for all $z \in \varrho\left(A^{*}\right) \backslash \mathrm{i} \mathbb{R}$ and thus, by continuity, for all $z \in \varrho\left(A^{*}\right)$.

Secondly, we use induction on $n \in \mathbb{N}$ to show that $\mathcal{N} \cap \operatorname{ker}\left(A^{*}-\lambda\right)^{n}=\{0\}$ for all $\lambda \in \sigma_{p}\left(A^{*}\right)$. The case $n=0$ is trivial. For $n \geq 1$, let $x \in \mathcal{N} \cap \operatorname{ker}\left(A^{*}-\lambda\right)^{n}$ and set $y:=\left(A^{*}-\lambda\right) x$. Since $A$ was assumed to have compact resolvent, the subspace $\mathcal{N} \cap \operatorname{ker}\left(A^{*}-\lambda\right)^{n}$ has finite dimension; by the first part of the proof, it is invariant under $\left(A^{*}-z\right)^{-1}$ and hence also under $A^{*}$. Therefore $y \in \mathcal{N} \cap \operatorname{ker}\left(A^{*}-\lambda\right)^{n-1}$. By induction, this yields $y=0$. Hence $x \in \operatorname{ker}\left(A^{*}-\lambda\right)$ and $0=B\left(A^{*}+\mathrm{i} t\right)^{-1} x=(\lambda+\mathrm{i} t)^{-1} B x$; thus $B x=0$. From (iv), we then obtain $x=0$.

Now let $\lambda \in \sigma_{p}(A)$ be arbitrary and let $P$ be the Riesz projection onto the corresponding generalised eigenspace of $A$. Then the Riesz projection corresponding to the eigenvalue $\bar{\lambda}$ of $A^{*}$ is given by

$$
P^{*}=\frac{\mathrm{i}}{2 \pi} \int_{\partial B_{\varepsilon}(\bar{\lambda})}\left(A^{*}-z\right)^{-1} \mathrm{~d} z
$$

with $\varepsilon>0$ such that $\overline{B_{\varepsilon}(\bar{\lambda})} \backslash\{\bar{\lambda}\} \subset \varrho\left(A^{*}\right)$. Since $\mathcal{N}$ is $\left(A^{*}-z\right)^{-1}$-invariant and closed, it is also invariant under $P^{*}$. Moreover, $\mathcal{R}\left(P^{*}\right)=\operatorname{ker}\left(A^{*}-\bar{\lambda}\right)^{n}$ for some $n \in \mathbb{N}$. For $x \in \mathcal{N}$, we obtain $P^{*} x \in \mathcal{N} \cap \operatorname{ker}\left(A^{*}-\bar{\lambda}\right)^{n}$ and so $P^{*} x=0$, i.e. $x \perp \mathcal{R}(P)$. Since $\lambda \in \sigma_{p}(A)$ was arbitrary, $x$ is orthogonal to the system of generalised eigenvectors of $A$, which was assumed to be complete, hence $x=0$. 
REMARK 5.7. If, in addition to being sectorially dichotomous, $A$ generates a strongly continuous semigroup and $B$ is bounded, then (25) is equivalent to the approximate controllability of the pair $(A, B)$, compare [13, §4.1].

REMARK 5.8. There is a second Riccati equation corresponding to the Hamiltonian $T$ : A linear operator $Y$ in the Hilbert space $H$ is a solution of the Riccati equation

$$
\left(A Y+Y\left(A^{*}-C Y\right)+B\right) v=0, \quad v \in \mathcal{D}\left(A^{*}\right) \cap Y^{-1} \mathcal{D}(A),
$$

if and only if the "inverse" graph subspace

$$
G_{\text {inv }}(Y):=\left\{\left(\begin{array}{c}
Y v \\
v
\end{array}\right) \mid v \in \mathcal{D}(Y)\right\}
$$

is $T$-invariant. The Riccati equations (24) and (27) are dual to each other in the following sense: $G_{\text {inv }}(Y)$ is $T$-invariant if and only if $G(Y)$ is invariant under the transformed Hamiltonian

$$
\widetilde{T}=\left(\begin{array}{ll}
0 & I \\
I & 0
\end{array}\right)\left(\begin{array}{cc}
A & B \\
C & -A^{*}
\end{array}\right)\left(\begin{array}{ll}
0 & I \\
I & 0
\end{array}\right)=\left(\begin{array}{cc}
-A^{*} & C \\
B & A
\end{array}\right) .
$$

For example, the dual version of Theorem 5.3 states that if, instead of (25),

$$
\bigcap_{t \in \mathbb{R}} \operatorname{ker} C(A-\mathrm{i} t)^{-1}=\{0\}
$$

holds, then $V_{ \pm}=G_{\text {inv }}\left(Y_{ \pm}\right)$where $Y_{ \pm}$is a selfadjoint nonnegative/nonpositive solution of (27), and $\mathcal{D}\left(A^{*}\right) \cap Y_{ \pm}^{-1} \mathcal{D}(A)$ is a core for $Y_{ \pm}$.

\section{Bounded solutions of Riccati equations}

In this section, we consider Hamiltonians $T$ for which $A$ is a sectorial operator with angle $\theta<\pi / 2$. Then the spectra of the diagonal entries $A$ and $-A^{*}$ of $T$ lie in the sectors $\Sigma_{\theta}$ and $\Sigma_{-\theta}$ in the right and left half-plane, respectively.

We show that then the solution $X_{+}$of the Riccati equation in Theorem 5.3 is bounded and uniquely determined; if $-A$ is sectorial, then $X_{-}$is bounded and uniquely determined.

LEMMA 6.1. Let $T$ be a nonnegative diagonally p-dominant Hamiltonian with $p<1$ and let $A$ be sectorially dichotomous. If the linear operator $X: H \rightarrow H$ is bounded and $G(X)$ is invariant under $T$ and under $(T-z)^{-1}, z \in \varrho(T)$, then $X \mathcal{D}(A) \subset \mathcal{D}\left(A^{*}\right)$ and $X$ is a solution of the Riccati equation

$$
\left(A^{*} X+X A+X B X-C\right) u=0, \quad u \in \mathcal{D}(A) .
$$

Proof. We consider the isomorphism $\varphi$ and the projection $\mathrm{pr}_{1}$ given by

$$
\begin{aligned}
\varphi: H & \rightarrow G(X), \quad \operatorname{pr}_{1}: H \times H & \rightarrow H, \\
u & \mapsto(u, X u), \quad(u, v) & \mapsto u,
\end{aligned}
$$


which are related by $\varphi^{-1}=\left.\operatorname{pr}_{1}\right|_{G(X)}$. Using the decomposition $T=S+R$ from (22) into diagonal and off-diagonal part, we define the operators $E:=\operatorname{pr}_{1} T \varphi$ and $F:=\operatorname{pr}_{1} R \varphi$ on $H$, i.e.

$$
\begin{array}{ll}
\mathcal{D}(E)=\mathcal{D}(A) \cap X^{-1} \mathcal{D}\left(A^{*}\right), & E u=A u+B X u, \\
\mathcal{D}(F)=\mathcal{D}(C) \cap X^{-1} \mathcal{D}(B), & F u=B X u .
\end{array}
$$

By assumption, $\mathcal{D}(A) \subset \mathcal{D}(C), \mathcal{D}\left(A^{*}\right) \subset \mathcal{D}(B)$ so that $\mathcal{D}(E-F)=\mathcal{D}(E) \subset \mathcal{D}(A)$ and $(E-F) u=A u$; hence $E-F$ is a restriction of $A$. We aim to show that, in fact, $\mathcal{D}(E-F)=\mathcal{D}(A)$.

Since $G(X)$ is $T$-invariant, $E=\left.\varphi^{-1} T\right|_{G(X)} \varphi$ and hence $\varrho(E)=\varrho\left(\left.T\right|_{G(X)}\right)$. Furthermore, we have $\varrho(T) \subset \varrho\left(\left.T\right|_{G(X)}\right)$ since $G(X)$ is also $(T-z)^{-1}$-invariant. By Theorem 4.6, the operator $T$ is dichotomous and thus $i \mathbb{R} \subset \varrho(T) \subset \varrho(E)$. From $\left\|R(S-\mathrm{i} t)^{-1}\right\| \leq M /|t|^{1-p}$ with some $M>0$, see (16) and Lemma 4.2, and from

$$
\begin{aligned}
F(E-\mathrm{i} t)^{-1} & =\operatorname{pr}_{1} R \varphi \varphi^{-1}(T-\mathrm{i} t)^{-1} \varphi=\operatorname{pr}_{1} R(T-\mathrm{i} t)^{-1} \varphi \\
& =\operatorname{pr}_{1} R(S-\mathrm{i} t)^{-1}\left(I+R(S-\mathrm{i} t)^{-1}\right)^{-1} \varphi
\end{aligned}
$$

we see that $\left\|F(E-\mathrm{i} t)^{-1}\right\|<1$ for large $|t|$. Consequently, it $\in \varrho(E-F)$ for large $|t|$. Since it $\in \varrho(A)$ for all $t \in \mathbb{R}$ and $E-F$ is a restriction of $A$, this implies that $\mathcal{D}(A)=\mathcal{D}(E-F)=\mathcal{D}(A) \cap X^{-1} \mathcal{D}\left(A^{*}\right)$, i.e. $X \mathcal{D}(A) \subset \mathcal{D}\left(A^{*}\right)$. The Riccati equation (28) now follows from Lemma 5.1.

LEMMA 6.2. Let $T$ be a nonnegative diagonally p-dominant Hamiltonian with $p<1$. Let $A$ be sectorial with angle $\theta<\pi / 2$ and $0 \in \varrho(A)$. If $X$ is a bounded selfadjoint solution of (28) with $X \mathcal{D}(A) \subset \mathcal{D}\left(A^{*}\right)$, then there exists a constant $L=$ $L\left(A, p, c_{C}\right)$ depending only on $A, p$, and the p-subordination bound $c_{C}$ of $C$ to $A$ such that

$$
(X u \mid u) \leq L\|u\|^{2}, \quad u \in H
$$

in particular, $\|X\| \leq L$ if $X$ is nonnegative.

Proof. From (28) and since $T$, and thus $B$, is nonnegative, we obtain

$$
(A u \mid X u)+(X u \mid A u)=(C u \mid u)-(B X u \mid X u) \leq(C u \mid u), \quad u \in \mathcal{D}(A) .
$$

Hence for arbitrary $t \in \mathbb{R}$,

$$
2 \operatorname{Re}((A-\mathrm{i} t) u \mid X u) \leq(C u \mid u), \quad u \in \mathcal{D}(A) .
$$

Together with the $p$-subordinacy of $C$ to $A$, this implies that for arbitrary $v \in H$, letting $u:=(A-\mathrm{i} t)^{-1} v$,

$$
\begin{aligned}
2 \operatorname{Re}\left(v \mid X(A-\mathrm{i} t)^{-1} v\right) & \leq\left(C(A-\mathrm{i} t)^{-1} v \mid(A-\mathrm{i} t)^{-1} v\right) \\
& \leq\left\|C(A-\mathrm{i} t)^{-1}\right\|\left\|(A-\mathrm{i} t)^{-1}\right\|\|v\|^{2} \\
& \leq c_{C}\left\|(A-\mathrm{i} t)^{-1}\right\|^{2-p}\left\|A(A-\mathrm{i} t)^{-1}\right\|^{p}\|v\|^{2} .
\end{aligned}
$$


Lemma 2.12 applied to the sectorial operator $A$ (for which $P_{-}=0$ ) yields that

$$
\begin{aligned}
& \frac{1}{\pi} \int_{\mathbb{R}}^{\prime}(A-\mathrm{i} t)^{-1} v \mathrm{~d} t=v, \quad v \in V, \\
& \left\|(A-\mathrm{i} t)^{-1}\right\| \leq \frac{M}{|t|}, \quad t \in \mathbb{R} \backslash\{0\} ;
\end{aligned}
$$

in particular, $\left\|A(A-\mathrm{i} t)^{-1}\right\|$ is uniformly bounded in $t \in \mathbb{R}$. Altogether, we obtain

$$
\begin{aligned}
2 \pi(X v \mid v) & =2 \pi \operatorname{Re}(X v \mid v)=\int_{\mathbb{R}}^{\prime} 2 \operatorname{Re}\left(X(A-\mathrm{i} t)^{-1} v \mid v\right) \mathrm{d} t \\
& \leq c_{C}\left(\int_{\mathbb{R}}\left\|(A-\mathrm{i} t)^{-1}\right\|^{2-p} \mathrm{~d} t\right) \sup _{t \in \mathbb{R}}\left\|A(A-\mathrm{i} t)^{-1}\right\|^{p}\|v\|^{2} \\
& =: L\left(A, p, c_{C}\right)\|v\|^{2} .
\end{aligned}
$$

The following proposition is the crucial step in proving the boundedness of a solution of the Riccati equation (26) in the presence of unbounded $B$ and $C$.

PROPOSITION 6.3. For $r \in[0,1]$, let $X_{r}$ be linear operators on $H$ and $P_{r}$ projections on $H \times H$ such that $\mathcal{R}\left(P_{r}\right)=G\left(X_{r}\right)$. Suppose that

(i) $P_{r}$ depends continuously on $r$ in the uniform operator topology;

(ii) $X_{0}$ is bounded;

(iii) there exists $L>0$ so that for all $r \in[0,1]$, if $X_{r}$ is bounded, then $\left\|X_{r}\right\| \leq L$.

Then all $X_{r}, r \in[0,1]$, are bounded.

Proof. Let $J:=\left\{r \in[0,1] \mid X_{r}\right.$ bounded $\}$. Then by assumption (ii), $0 \in J$. We will show that $J$ is closed and open in the interval $[0,1]$ and hence equal to $[0,1]$.

Let $\left(r_{n}\right)_{n \in \mathbb{N}} \subset J, \lim _{n \rightarrow \infty} r_{n}=r$, and $u \in \mathcal{D}\left(X_{r}\right)$. Set

$$
x:=\left(\begin{array}{c}
u \\
X_{r} u
\end{array}\right), \quad P_{r_{n}} x=:\left(\begin{array}{c}
u_{n} \\
X_{r_{n}} u_{n}
\end{array}\right), \quad n \in \mathbb{N} .
$$

Then $\lim _{n \rightarrow \infty} P_{r_{n}} x=P_{r} x=x$, which implies that $u_{n} \rightarrow u$ and $X_{r_{n}} u_{n} \rightarrow X_{r} u$ as $n \rightarrow \infty$. By assumption (iii), we obtain

$$
\left\|X_{r} u\right\|=\lim _{n \rightarrow \infty}\left\|X_{r_{n}} u_{n}\right\| \leq L \lim _{n \rightarrow \infty}\left\|u_{n}\right\|=L\|u\|
$$

and hence $r \in J$. Therefore $J$ is closed.

Now suppose that $J$ is not open. Then there exist $r \in J$ and $\left(r_{n}\right)_{n \in \mathbb{N}} \subset[0,1] \backslash J$ such that $\lim _{n \rightarrow \infty} r_{n}=r$. So all $X_{r_{n}}$ are unbounded. Hence there are $u_{n} \in \mathcal{D}\left(X_{r_{n}}\right)$ with $\left\|u_{n}\right\| \leq 1 / n$ and $\left\|X_{r_{n}} u_{n}\right\|=1$. Set

$$
x_{n}:=\left(\begin{array}{c}
u_{n} \\
X_{r_{n}} u_{n}
\end{array}\right), \quad P_{r} x_{n}=:\left(\begin{array}{c}
w_{n} \\
X_{r} w_{n}
\end{array}\right), \quad n \in \mathbb{N} .
$$


Since $x_{n} \in G\left(X_{r_{n}}\right)=\mathcal{R}\left(P_{r_{n}}\right)$ for all $n \in \mathbb{N}$, we have $P_{r_{n}} x_{n}=x_{n}$ and

$$
\begin{aligned}
1 & =\left\|X_{r_{n}} u_{n}\right\| \leq\left\|X_{r_{n}} u_{n}-X_{r} w_{n}\right\|+\left\|X_{r}\right\|\left(\left\|w_{n}-u_{n}\right\|+\left\|u_{n}\right\|\right) \\
& \leq\left\|P_{r_{n}} x_{n}-P_{r} x_{n}\right\|+\left\|X_{r}\right\|\left(\left\|P_{r} x_{n}-P_{r_{n}} x_{n}\right\|+\left\|u_{n}\right\|\right) \\
& \leq\left(1+\left\|X_{r}\right\|\right)\left\|P_{r_{n}}-P_{r}\right\|\left\|x_{n}\right\|+\left\|X_{r}\right\|\left\|u_{n}\right\| .
\end{aligned}
$$

Since $\left\|x_{n}\right\|^{2}=\left\|u_{n}\right\|^{2}+\left\|X_{r_{n}} u_{n}\right\|^{2} \leq 1 / n^{2}+1$ and $P_{r_{n}} \rightarrow P_{r}, u_{n} \rightarrow 0$ as $n \rightarrow \infty$, this is a contradiction.

THEOREM 6.4. Let $T$ be a nonnegative diagonally p-dominant Hamiltonian with $p<1$. Suppose that $\mathrm{i} \mathbb{R} \subset \varrho(A)$ and assumption (25) holds, i.e.

$$
\bigcap_{t \in \mathbb{R}} \operatorname{ker}\left(B\left(A^{*}+\mathrm{i} t\right)^{-1}\right)=\{0\} .
$$

(i) If $A$ is sectorial with angle $\theta<\pi / 2$, then the nonnegative solution $X_{+}$of the Riccati equation (26) in Theorem 5.3 is bounded, satisfies $X_{+} \mathcal{D}(A) \subset \mathcal{D}\left(A^{*}\right)$ and hence

$$
\left(A^{*} X_{+}+X_{+} A+X_{+} B X_{+}-C\right) u=0, \quad u \in \mathcal{D}(A) .
$$

Moreover, $X_{+}$is the uniquely determined bounded nonnegative operator such that $X_{+} \mathcal{D}(A) \subset \mathcal{D}\left(A^{*}\right)$ and (30) hold.

(ii) If $-A$ is sectorial with angle $\theta<\pi / 2$, then the nonpositive solution $X_{-}$of the Riccati equation (26) in Theorem 5.3 is bounded, satisfies $X_{-} \mathcal{D}(A) \subset \mathcal{D}\left(A^{*}\right)$, and hence

$$
\left(A^{*} X_{-}+X_{-} A+X_{-} B X_{-}-C\right) u=0, \quad u \in \mathcal{D}(A) .
$$

Moreover, $X_{-}$is the uniquely determined bounded nonpositive operator such that $X_{-} \mathcal{D}(A) \subset \mathcal{D}\left(A^{*}\right)$ and (31) hold.

Proof. Suppose that $A$ is sectorial. We consider the family of operators $T_{r}=S+r R$, $r \in[0,1]$, where $S, R$ are the diagonal and off-diagonal part of $T$, respectively, as in (22). By Theorem 4.6, each $T_{r}$ is dichotomous and the corresponding projections $P_{r,+}$ and $P_{r,-}$ satisfy

$$
2 P_{r,+} x-x=P_{r,+} x-P_{r,-} x=\frac{1}{\pi} \int_{\mathbb{R}}^{\prime}\left(T_{r}-\mathrm{i} t\right)^{-1} x \mathrm{~d} t, \quad x \in H \times H .
$$

For $r>0$, Theorem 5.3 applies to $T_{r}$ since $\operatorname{ker}\left(B\left(A^{*}+\mathrm{i} t\right)^{-1}\right)=\operatorname{ker}\left(r B\left(A^{*}+\mathrm{i} t\right)^{-1}\right)$ if $r>0$. Hence there are nonnegative selfadjoint operators $X_{r}, r>0$, such that $\mathcal{R}\left(P_{r,+}\right)=G\left(X_{r}\right)$; in particular, $X_{1}=X_{+}$. For $r=0$ we have $T_{0}=S$ and $\mathcal{R}\left(P_{0,+}\right)=$ $H \times\{0\}=G\left(X_{0}\right)$ where $X_{0}=0$, see also Lemma 2.12. If we set $P_{r}:=P_{r,+}$, then we obtain, for $r, s \in[0,1]$,

$$
P_{r} x-P_{s} x=\frac{1}{2 \pi} \int_{\mathbb{R}}^{\prime}\left((S+r R-\mathrm{i} t)^{-1}-(S+s R-\mathrm{i} t)^{-1}\right) x \mathrm{~d} t, \quad x \in H \times H .
$$


Since $\left\|R(S-\mathrm{i} t)^{-1}\right\| \leq M^{\prime} /|t|^{1-p}$, see (16), and $r \in[0,1]$, a Neumann series argument yields $\left\|\left(I+r R(S-\mathrm{i} t)^{-1}\right)^{-1}\right\| \leq 2$ for $|t| \geq t_{0}$, where the constant $t_{0}>0$ is independent of $r$. Using $\left\|(S-\mathrm{i} t)^{-1}\right\| \leq M /|t|$ and

$$
\begin{aligned}
& (S+r R-\mathrm{i} t)^{-1}-(S+s R-\mathrm{i} t)^{-1} \\
& =(S+s R-\mathrm{i} t)^{-1}(s-r) R(S+r R-\mathrm{i} t)^{-1} \\
& =(S-\mathrm{i} t)^{-1}\left(I+s R(S-\mathrm{i} t)^{-1}\right)^{-1}(s-r) R(S-\mathrm{i} t)^{-1}\left(I+r R(S-\mathrm{i} t)^{-1}\right)^{-1},
\end{aligned}
$$

we find that

$$
\left\|(S+r R-\mathrm{i} t)^{-1}-(S+s R-\mathrm{i} t)^{-1}\right\| \leq|s-r| \cdot \frac{4 M M^{\prime}}{|t|^{2-p}} \text { for }|t| \geq t_{0} .
$$

The identity

$$
(S+s R-\mathrm{i} \tilde{t})^{-1}=(S+r R-\mathrm{i} t)^{-1}\left(I+((s-r) R+\mathrm{i} t-\mathrm{i} \tilde{t})(S+r R-\mathrm{i} t)^{-1}\right)^{-1}
$$

implies that the mapping $(r, t) \mapsto(S+r R-\mathrm{i} t)^{-1}$ is continuous in the operator norm topology. On the compact set $\left\{(r, t) \in \mathbb{R}^{2}|r \in[0,1]| t \mid, \leq t_{0}\right\}$ it is thus uniformly continuous. Hence for $\varepsilon>0$, there exists $\delta>0$ such that

$$
\left\|(S+r R-\mathrm{i} t)^{-1}-(S+s R-\mathrm{i} t)^{-1}\right\|<\varepsilon \text { for }|t| \leq t_{0},|s-r|<\delta .
$$

From (32), (33), and (34) we now obtain, for $|s-r|<\delta$,

$$
\left\|P_{r}-P_{s}\right\| \leq \frac{1}{2 \pi}\left(2 t_{0} \varepsilon+|s-r| \int_{|t| \geq t_{0}} \frac{4 M M^{\prime}}{|t|^{2-p}} \mathrm{~d} t\right) .
$$

Consequently, the mapping $r \mapsto P_{r}$ is continuous. Since $G\left(X_{r}\right)=\mathcal{R}\left(P_{r}\right)$ are invariant under $T_{r}$ and its resolvent, Lemmas 6.1, 6.2 apply; using $c_{r} \leq c_{C}$ for $r \in[0,1]$, we obtain a constant $L=L\left(A, p, c_{C}\right)>0$ independent of $r \in[0,1]$ such that if $X_{r}$ is bounded, then $\left\|X_{r}\right\| \leq L$. Hence Proposition 6.3 yields that all $X_{r}$ are bounded.

To show the uniqueness of $X_{+}$, suppose that $X$ is another bounded nonnegative solution of (30) with $X \mathcal{D}(A) \subset \mathcal{D}\left(A^{*}\right)$. Let $\varphi: H \rightarrow G(X), \varphi u=(u, X u)$ be the isomorphism defined in (29) in the proof of Lemma 6.1. Our assumptions on $X$ imply that $G(X)$ is $T$-invariant and that $\varphi^{-1} T \varphi=A+B X$. Consequently, $\sigma\left(\left.T\right|_{G(X)}\right)=$ $\sigma(A+B X)$.

The operator $A^{*} X$ is closable and $\mathcal{D}(A) \subset \mathcal{D}\left(A^{*} X\right)$, hence $A^{*} X$ is $A$-bounded. Thus since $0 \in \varrho(A)$, there exists $c>0$ such that $\left\|A^{*} X u\right\| \leq c\|A u\|$ for $u \in \mathcal{D}(A)$. Together with the $p$-subordination property of $B$ to $A^{*}$, we then find

$$
\|B X u\| \leq c_{B}\|X u\|^{1-p}\left\|A^{*} X u\right\|^{p} \leq c_{B} c^{p}\|X\|^{1-p}\|u\|^{1-p}\|A u\|^{p}, \quad u \in \mathcal{D}(A),
$$

i.e. $B X$ is $p$-subordinate to $A$. Since $A$ is sectorial with angle $\theta<\pi / 2$, Remark 3.8 implies that $A+B X$ is sectorial with some angle $\theta^{\prime} \in[\theta, \pi / 2[$ and radius $r>0$, in particular,

$$
\sigma(A+B X) \subset \Sigma_{\theta^{\prime}} \cup \overline{B_{r}(0)}
$$


We will now show that $\sigma(A+B X)$ is, in fact, contained in the open right half-plane; this shows, in particular, that $A+B X$ is sectorial with radius 0 . In view of (35), it is sufficient to prove that $\lambda \in r(A+B X)$ whenever $\operatorname{Re} \lambda \leq 0$. Suppose, to the contrary, that $\lambda \in \mathbb{C}$ with $\operatorname{Re} \lambda \leq 0$ and there exist $u_{n} \in \mathcal{D}(A),\left\|u_{n}\right\|=1$, with

$$
\lim _{n \rightarrow \infty}(A+B X-\lambda) u_{n}=0
$$

This implies

$$
\lim _{n \rightarrow \infty}\left((A+B X-\lambda) u_{n} \mid X u_{n}\right)=0 .
$$

Moreover, the Riccati equation (30) for $X$ yields that

$$
\begin{aligned}
\operatorname{Re}((A+B X) u \mid X u) & =\frac{1}{2}((A u \mid X u)+(X u \mid A u))+(B X u \mid X u) \\
& =\frac{1}{2}((C u \mid u)+(B X u \mid X u)), \quad u \in \mathcal{D}(A) .
\end{aligned}
$$

Combining (37) and (38) and using $B, C \geq 0$, we find

$$
\begin{aligned}
\limsup _{n \rightarrow \infty}\left((\operatorname{Re} \lambda) \cdot\left(X u_{n} \mid u_{n}\right)\right) & =\limsup _{n \rightarrow \infty} \operatorname{Re}\left((A+B X) u_{n} \mid X u_{n}\right) \\
& =\limsup _{n \rightarrow \infty} \frac{1}{2}\left(\left(C u_{n} \mid u_{n}\right)+\left(B X u_{n} \mid X u_{n}\right)\right) \\
& \geq \frac{1}{2} \limsup _{n \rightarrow \infty}\left(B X u_{n} \mid X u_{n}\right) \geq 0 .
\end{aligned}
$$

On the other hand, $X \geq 0$ and $\operatorname{Re} \lambda \leq 0$ imply $\lim _{\sup } \rightarrow \infty\left((\operatorname{Re} \lambda) \cdot\left(X u_{n} \mid u_{n}\right)\right) \leq 0$; consequently, $\lim _{n \rightarrow \infty}\left\|B^{1 / 2} X u_{n}\right\|=0$. This, the fact that $B^{1 / 2}\left(A^{*}-\bar{\lambda}\right)^{-1}$ and thus $(A-\lambda)^{-1} B^{1 / 2}$ is bounded, and (36) now yield that

$$
\begin{aligned}
\lim _{n \rightarrow \infty} u_{n} & =\lim _{n \rightarrow \infty}\left(u_{n}+(A-\lambda)^{-1} B^{1 / 2} \cdot B^{1 / 2} X u_{n}\right) \\
& =(A-\lambda)^{-1} \lim _{n \rightarrow \infty}(A+B X-\lambda) u_{n}=0,
\end{aligned}
$$

a contradiction to $\left\|u_{n}\right\|=1$.

Since $A+B X$ is sectorial of angle $<\pi / 2$ and its spectrum is contained in the open right half-plane, $(A+B X-\lambda)^{-1}$ and hence $\left(\left.T\right|_{G(X)}-\lambda\right)^{-1}$ are uniformly bounded on $\mathbb{C}_{-}$. Therefore $(T-\lambda)^{-1}(u, X u)$ extends to a bounded analytic function on $\mathbb{C}_{-}$which, in turn, implies that $(u, X u)$ belongs to the spectral subspace $V_{+}=G\left(X_{+}\right)$of $T$, see [9, Sect. 2 and Theorem 3.1]. This proves that $G(X) \subset G\left(X_{+}\right)$and hence $X=X_{+}$since the bounded operators $X$ and $X_{+}$are both defined on the dense subset $\mathcal{D}(A) \subset H$.

The case when $-A$ is sectorial is a consequence of the first case since $-X_{-}$is nonnegative and a solution of the Riccati equation corresponding to

$$
\left(\begin{array}{cc}
-A & B \\
C & A^{*}
\end{array}\right) .
$$


REMARK 6.5. In [1, Section 7] the existence and uniqueness of solutions of Riccati equations was proved under the weaker assumption that $B, C$ are uniformly accretive, but only in the case where all entries $A, B$, and $C$ are bounded and using a different approach.

\section{Examples}

To illustrate the results of the previous sections, we consider three examples of Hamiltonians involving partial differential and multiplication operators. None of these examples is covered by the earlier results in [11,21,24,41,42].

In all examples, $B$ and $C$ are unbounded and hence $[11,21,24]$ cannot be applied. Moreover, $B$ and $C$ do not map into an extrapolation space of $H$ and thus do not fit into the setting of [42]. In the first example, the operator $A$ has continuous spectrum and hence no Riesz basis of generalised eigenvectors exists as required in [41].

EXAMPLE 7.1. We consider the Hilbert space $H=L^{2}\left(\mathbb{R}^{n}\right)$ and the operators

$$
\begin{array}{ll}
A u=(-\Delta+\varepsilon) u, & \mathcal{D}(A)=W^{2,2}\left(\mathbb{R}^{n}\right), \\
B u=g_{1} u, & \mathcal{D}(B)=\left\{u \in H \mid g_{1} u \in H\right\}, \\
C u=g_{2} u, & \mathcal{D}(C)=\left\{u \in H \mid g_{2} u \in H\right\},
\end{array}
$$

with $\varepsilon>0$ and nonnegative locally integrable functions $g_{1}, g_{2}: \mathbb{R}^{n} \rightarrow \mathbb{R}$. Suppose, in addition, that $g_{1}$ is positive almost everywhere and that $g_{1}, g_{2}$ satisfy estimates

$$
\int_{B_{r}\left(x_{0}\right)}\left|g_{j}(x)\right|^{2} \mathrm{~d} x \leq c r^{s}, \quad x_{0} \in \mathbb{R}^{n}, 0<r<1, j=1,2,
$$

with constants $c>0$ and $s \in[0, n]$ such that $s>n-4$; e.g. one could choose $g(x)=|x|^{-q}$ with $0<q<\min \{2, n / 2\}$ and $s=n-2 q$.

The operator $A$ is positive and selfadjoint, $0 \in \varrho(A)$, and the a priori estimate

$$
\|u\|_{W^{2,2}\left(\mathbb{R}^{n}\right)} \leq c_{0}\|A u\|, \quad u \in \mathcal{D}(A)
$$

can easily be verified by Fourier transformation. The multiplication operators $B$ and $C$ are selfadjoint, $B$ is positive, and $C$ is nonnegative. By Lemma 3.3, $B$ and $C$ are $p$-subordinate to $A$ with $p=\frac{1}{4}(n-s)<1$. We can thus apply our results to the Hamiltonian

$$
T=\left(\begin{array}{cc}
A & B \\
C & -A
\end{array}\right)
$$

note that condition (25) holds because ker $B=\{0\}$.

Hence Theorem 6.4 yields the existence and uniqueness of a bounded nonnegative selfadjoint solution $X_{+}$of the Riccati equation

$$
\left(A X_{+}+X_{+} A+X_{+} B X_{+}-C\right) u=0, \quad u \in \mathcal{D}(A)
$$


Theorem 5.3 yields the existence of a nonpositive selfadjoint solution $X_{-}$of the Riccati equation

$$
\left(A X_{-}+X_{-}\left(A+B X_{-}\right)-C\right) u=0, \quad u \in \mathcal{D}(A) \cap X_{-}^{-1} \mathcal{D}(A) .
$$

In the next two examples, $A$, and hence also $T$, has compact resolvent and pure point spectrum. However, $A$ is not normal as required in the known existence results for Riesz bases of generalised eigenvectors, e.g. [28, Theorem 6.12], [40, Theorem 6.1], and thus [41] cannot be applied.

EXAMPLE 7.2. Let $\Omega \subset \mathbb{R}^{n}$ be open and bounded with smooth boundary $\partial \Omega$ such that no point of $\partial \Omega$ belongs to the interior of $\bar{\Omega}$. Let $H=L^{2}(\Omega)$ and consider the operators

$$
\begin{aligned}
& A u=\Delta^{2} u, \quad \mathcal{D}(A)=\left\{u \in W^{4,2}(\Omega) \mid u=\Delta u+f \partial_{\nu} u=0 \text { on } \partial \Omega\right\}, \\
& B u=-\sum_{j, k=1}^{n} \partial_{j}\left(g_{j k} \partial_{k} u\right)+g_{0} u, \quad \mathcal{D}(B)=W^{2,2}(\Omega) \cap W_{0}^{1,2}(\Omega), \\
& C u=-\sum_{j, k=1}^{n} \partial_{j}\left(h_{j k} \partial_{k} u\right)+h_{0} u, \quad \mathcal{D}(C)=W^{2,2}(\Omega) \cap W_{0}^{1,2}(\Omega),
\end{aligned}
$$

where $f \in C^{\infty}(\partial \Omega)$ with $\operatorname{Re} f \geq 0, g_{j k}, h_{j k}, g_{0}, h_{0} \in C^{\infty}(\Omega), g_{0}, h_{0} \geq 0$, and the matrices $\left(g_{j k}\right)_{j, k=1 \ldots n}$ and $\left(h_{j k}\right)_{j, k=1 \ldots n}$ are positive definite and positive semidefinite, respectively, almost everywhere on $\Omega$. The outward normal derivative is $\partial_{v}$.

From the theory of elliptic partial differential operators, see e.g. [27], it follows that $B$ and $C$ are selfadjoint, $B$ is positive, and $C$ is nonnegative. The operator $A$ is closed and its adjoint is given by

$$
A^{*} u=\Delta^{2} u, \quad \mathcal{D}\left(A^{*}\right)=\left\{u \in W^{4,2}(\Omega) \mid u=\Delta u+\bar{f} \partial_{\nu} u=0 \text { on } \partial \Omega\right\} ;
$$

note that $A$ is not selfadjoint (not even normal) if $\operatorname{Im} f \neq 0$. Integration by parts shows that

$$
(A u \mid u)=\int_{\Omega}|\Delta u|^{2} \mathrm{~d} x+\int_{\partial \Omega} f\left|\partial_{\nu} u\right|^{2} \mathrm{~d} \sigma, \quad u \in \mathcal{D}(A) .
$$

Consequently, there exist $c_{0}, c_{1}>0$ such that for $u \in \mathcal{D}(A)$

$$
\operatorname{Re}(A u \mid u) \geq\|\Delta u\|^{2} \geq c_{0}\|u\|_{W^{2,2}(\Omega)}^{2}, \quad|\operatorname{Im}(A u \mid u)| \leq c_{1}\|u\|_{W^{2,2}(\Omega)}^{2} .
$$

This implies that ker $A=\{0\}$, that $\mathcal{R}(A)$ is closed, and that the numerical range $W(A)$ is contained in a sector $\Sigma_{\theta}$, see (4), more precisely,

$$
W(A) \subset\left\{z \in \Sigma_{\theta} \mid \operatorname{Re} z \geq c_{0}\right\} \text { with } \theta=\arctan \left(c_{1} / c_{0}\right) .
$$

Since (39) (with $f$ replaced by $\bar{f}$ ) and (40) also hold for $A^{*}$, this yields $\mathcal{R}(A)^{\perp}=$ $\operatorname{ker} A^{*}=\{0\}$ and thus $0 \in \varrho(A)$. In view of Remark 2.9 (iii), we obtain that $A$ is sectorial with angle $\theta$. Finally, (40) also implies

$$
\|u\|_{W^{2,2}(\Omega)} \leq c_{0}^{-1}\|u\|^{1 / 2}\|A u\|^{1 / 2}, \quad u \in \mathcal{D}(A),
$$


and the same with $A$ replaced by $A^{*}$. Consequently, $B$ and $C$ are $\frac{1}{2}$-subordinate to $A^{*}$ and $A$, respectively. Moreover, $\operatorname{ker} B=\{0\}$ since $B$ is positive and thus assumption (25) is satisfied.

Hence Theorem 6.4 shows that there exists a unique bounded nonnegative selfadjoint solution $X_{+}$of the Riccati equation

$$
\left(A^{*} X_{+}+X_{+} A+X_{+} B X_{+}-C\right) u=0, \quad u \in \mathcal{D}(A)
$$

Theorem 5.3 shows that there exists a nonpositive selfadjoint solution $X_{-}$of the Riccati equation

$$
\left(A^{*} X_{-}+X_{-}\left(A+B X_{-}\right)-C\right) u=0, \quad u \in \mathcal{D}(A) \cap X_{-}^{-1} \mathcal{D}\left(A^{*}\right)
$$

In our final example, we consider a Riccati equation with coefficients $\widetilde{A}, \widetilde{B}$, and $\widetilde{C}$ such that $\widetilde{A}$ is sectorially dichotomous, but neither $\widetilde{A}$ nor $-\widetilde{A}$ are sectorial. Hence Theorem 6.4 does not apply and both solutions $X_{ \pm}$will be unbounded in general.

EXAMPLE 7.3. Let $\Omega \subset \mathbb{R}^{n}$ and the operators $A, B, C$ in $L^{2}(\Omega)$ be as in the previous example. Consider the block operator matrices

$$
\widetilde{A}=\left(\begin{array}{cc}
A & 0 \\
0 & -A^{*}
\end{array}\right), \quad \widetilde{B}=\left(\begin{array}{cc}
B & \beta B \\
\beta B & B
\end{array}\right), \quad \widetilde{C}=\left(\begin{array}{cc}
C & \gamma C \\
\gamma C & C
\end{array}\right) \quad \text { in } L^{2}(\Omega) \times L^{2}(\Omega)
$$

where $\beta \in[0,1[, \gamma \in[0,1]$. Then $\widetilde{A}$ is sectorially dichotomous, $\widetilde{B}, \widetilde{C}$ are symmetric, $\widetilde{B}$ is positive since $\beta<1, \widetilde{C}$ is nonnegative, and $\widetilde{B}, \widetilde{C}$ are $\frac{1}{2}$-subordinate to $\widetilde{A}^{*}, \widetilde{A}$, respectively. By Theorem 5.3, the Riccati equation

$$
\left(\widetilde{A}^{*} X_{ \pm}+X_{ \pm}\left(\widetilde{A}+\widetilde{B} X_{ \pm}\right)-\widetilde{C}\right) u=0, \quad u \in \mathcal{D}(\widetilde{A}) \cap X_{ \pm}^{-1} \mathcal{D}\left(\widetilde{A}^{*}\right)
$$

has two selfadjoint solutions $X_{ \pm}$, where $X_{+}$is nonnegative and $X_{-}$is nonpositive, both of which are unbounded in general.

\section{Acknowledgments}

The support for this work by Deutsche Forschungsgemeinschaft (DFG), Grant TR 368/6-2, and Schweizerischer Nationalfonds (SNF), Grant 200021-119826/1, is gratefully acknowledged. We would also like to thank the referee very much for useful comments.

\section{REFERENCES}

[1] V. Adamjan, H. Langer, C. Tretter. Existence and uniqueness of contractive solutions of some Riccati equations. J. Funct. Anal., 179(2) (2001), 448-473.

[2] R. A. Adams, J. J. F. Fournier. Sobolev spaces, volume 140 of Pure and Applied Mathematics. Elsevier/Academic Press, Amsterdam, second edition, 2003.

[3] N. I. Akhiezer, I. M. Glazman. Theory of Linear Operators in Hilbert Space. Dover Publications Inc., New York, 1993. 
[4] W. Arendt, S. Bu. Sums of bisectorial operators and applications. Integral Equations Operator Theory, 52(3) (2005), 299-321.

[5] W. Arendt, M. Duelli. Maximal $L^{p}$-regularity for parabolic and elliptic equations on the line. J. Evol. Equ., 6(4) (2006), 773-790.

[6] T. Y. Azizov, A. Dijksma, I. V. Gridneva. Conditional reducibility of certain unbounded nonnegative Hamiltonian operator functions. Integral Equations Operator Theory, 73(2) (2012), 273-303.

[7] T. Y. Azizov, I. S. Iokhvidov. Linear Operators in Spaces With an Indefinite Metric. John Wiley \& Sons, Chichester, 1989.

[8] H. Bart, I. Gohberg, M. A. Kaashoek. Minimal factorization of matrix and operator functions, volume 1 of Operator Theory: Advances and Applications. Birkhäuser Verlag, Basel, 1979.

[9] H. Bart, I. Gohberg, M. A. Kaashoek. Wiener-Hopf factorization, inverse Fourier transforms and exponentially dichotomous operators. J. Funct. Anal., 68(1) (1986), 1-42.

[10] S. Bittanti, A. J. Laub, J. C. Willems, editors. The Riccati equation. Communications and Control Engineering Series. Springer-Verlag, Berlin, 1991.

[11] P. Bubák, C. V. M. van der Mee, A. C. M. Ran. Approximation of solutions of Riccati equations. SIAM J. Control Optim., 44(4) (2005), 1419-1435.

[12] J.-C. Cuenin, C. Tretter. Spectral perturbations of self-adjoint operators with spectral gaps. Submitted, (2013).

[13] R. F. Curtain, H. J. Zwart. An Introduction to Infinite Dimensional Linear Systems Theory. Springer, New York, 1995.

[14] A. Dijksma, H. S. V. de Snoo. Symmetric and selfadjoint relations in Krĕn spaces. I. volume 24 of Oper. Theory Adv. Appl., pages 145-166. Birkhäuser, Basel, 1987.

[15] K.-J. Engel, R. Nagel. One-Parameter Semigroups for Linear Evolution Equations. Springer, New York, 2000.

[16] I. Gohberg, S. Goldberg, M. A. Kaashoek. Classes of linear operators. Vol. I, volume 49 of Operator Theory: Advances and Applications. Birkhäuser, Basel, 1990.

[17] T. Kato. Perturbation Theory for Linear Operators. Springer, Berlin, 1980.

[18] V. Kostrykin, K. A. Makarov, A. K. Motovilov. Existence and uniqueness of solutions to the operator Riccati equation. A geometric approach. volume 327 of Contemp. Math., pages 181-198. Amer. Math. Soc., Providence, 2003.

[19] M. G. Kreun. Introduction to the geometry of indefinite $J$-spaces and to the theory of operators in those spaces. In Second Math. Summer School, Part I (Russian), pages 15-92. Naukova Dumka, Kiev, 1965. English transl. in Amer. Math. Soc. Transl. (2), 93 (1970), 103-176.

[20] S. G. Krĕ̌n. Linear Differential Equations in Banach Space. Amer. Math. Soc., Providence, 1971.

[21] C. R. Kuiper, H. J. Zwart. Connections between the algebraic Riccati equation and the Hamiltonian for Riesz-spectral systems. J. Math. Systems Estim. Control, 6(4) (1996), 1-48.

[22] P. Lancaster, L. Rodman. Algebraic Riccati Equations. Oxford University Press, Oxford, 1995.

[23] H. Langer, A. C. M. Ran, D. Temme. Nonnegative solutions of algebraic Riccati equations. Linear Algebra Appl., 261 (1997), 317-352.

[24] H. Langer, A. C. M. Ran, B. A. van de Rotten. Invariant subspaces of infinite dimensional Hamiltonians and solutions of the corresponding Riccati equations. volume 130 of Oper. Theory Adv. Appl., pages 235-254. Birkhäuser, Basel, 2002.

[25] H. Langer, C. Tretter. Diagonalization of certain block operator matrices and applications to Dirac operators. volume 122 of Oper. Theory Adv. Appl., pages 331-358. Birkhäuser, Basel, 2001.

[26] I. Lasiecka, R. Triggiani. Control Theory for Partial Differential Equations: Continuous and Approximation Theorems. I: Abstract Parabolic Systems. Cambridge University Press, 2000.

[27] J.-L. Lions, E. Magenes. Non-homogeneous boundary value problems and applications. Vol. I. Springer-Verlag, New York, 1972.

[28] A. S. Markus. Introduction to the Spectral Theory of Polynomial Operator Pencils. Amer. Math. Soc., Providence, 1988.

[29] V. Maz'ya. Sobolev spaces with applications to elliptic partial differential equations, volume 342 of Grundlehren der Mathematischen Wissenschaften. Springer, Heidelberg, augmented edition, 2011.

[30] A. McIntosh, A. Yagi. Operators of type $\omega$ without a bounded $H_{\infty}$ functional calculus. In Miniconference on Operators in Analysis (Sydney, 1989), volume 24 of Proc. Centre Math. Anal. Austral. Nat. Univ., pages 159-172. Austral. Nat. Univ., Canberra, 1990. 
[31] M. R. Opmeer, R. F. Curtain. New Riccati equations for well-posed linear systems. Systems Control Lett., 52(5) (2004), 339-347.

[32] O. Staffans. Well-posed linear systems, volume 103 of Encyclopedia of Mathematics and its Applications. Cambridge University Press, Cambridge, 2005.

[33] C. Tretter. Spectral issues for block operator matrices. In Differential equations and mathematical physics (Birmingham, AL, 1999), volume 16 of AMS/IP Stud. Adv. Math., pages 407-423. Amer. Math. Soc., Providence, 2000.

[34] C. Tretter. Spectral Theory of Block Operator Matrices and Applications. Imperial College Press, London, 2008.

[35] C. Tretter. Spectral inclusion for unbounded block operator matrices. J. Funct. Anal., 256(11) (2009), 3806-3829.

[36] H. Triebel. Interpolation theory, function spaces, differential operators. Johann Ambrosius Barth Verlag, Heidelberg, Leipzig, 1995.

[37] C. van der Mee. Exponentially dichotomous operators and applications, volume 182 of Operator Theory: Advances and Applications. Birkhäuser Verlag, Basel, 2008.

[38] J. Weidmann. Linear operators in Hilbert spaces, volume 68 of Graduate Texts in Mathematics. Springer-Verlag, New York, 1980.

[39] M. Weiss, G. Weiss. Optimal control of stable weakly regular linear systems. Math. Control Signals Systems, 10(4) (1997), 287-330.

[40] C. Wyss. Riesz bases for p-subordinate perturbations of normal operators. J. Funct. Anal., 258(1) (2010), 208-240.

[41] C. Wyss. Hamiltonians with Riesz bases of generalised eigenvectors and Riccati equations. Indiana Univ. Math. J., 60 (2011), 1723-1766.

[42] C. Wyss, B. Jacob, H. J. Zwart. Hamiltonians and Riccati equations for linear systems with unbounded control and observation operators. SIAM J. Control Optim., 50 (2012), 1518-1547.

\author{
C. Tretter \\ Institute of Mathematics, \\ University of Bern, \\ Sidlerstrasse 5, 3012 Bern, Switzerland \\ E-mail: tretter@math.unibe.ch \\ C. Wyss \\ Department of Mathematics and Informatics, \\ University of Wuppertal, \\ Gaußstraße 20, 42097 Wuppertal, Germany \\ E-mail:wyss@math.uni-wuppertal.de
}

